\title{
A Secure Anonymous D2D Mutual Authentication and Key Agreement Protocol for loT Environments
}

Rahman Hajian

Islamic Azad University South Tehran Branch

Abbas Haghighat

Islamic Azad University of Safashahr

S.Hossein Erfani ( $\boldsymbol{\sim}$ h_erfani@azad.ac.ir)

Islamic Azad University https://orcid.org/0000-0002-7893-4191

\section{Research Article}

Keywords: Internet of Things (IoT), Device to Device (D2D) authentication, Key agreement, Key Compromise Impersonation (KCl) attack.

Posted Date: June 16th, 2021

DOI: https://doi.org/10.21203/rs.3.rs-223151/v1

License: (9) This work is licensed under a Creative Commons Attribution 4.0 International License.

Read Full License 


\title{
A Secure Anonymous D2D Mutual Authentication and Key Agreement Protocol for IoT Environments
}

\author{
${ }^{1}$ R. Hajian (1), ${ }^{2}$ A. Haghighat, ${ }^{* 3}$ S. H. Erfani ${ }^{(1)}$ \\ ${ }^{1}$ Department of Information Technology Engineering, South Tehran Branch, Islamic Azad University, Tehran, Iran \\ ${ }^{2}$ Department of Electrical and Computer Engineering, Safashahr Branch, Islamic Azad University, Safashahr, Iran \\ ${ }^{3}$ Department of Computer Engineering, South Tehran Branch, Islamic Azad University, Tehran, Iran \\ *Corresponding: $\underline{\text { h_erfani@azad.ac.ir }}$
}

\begin{abstract}
Internet of Things (IoT) is a developing technology in our time that is prone to security problems as it uses wireless and shared networks. A challenging scenario in IoT environments is Device-to-Device (D2D) communication that an authentication server as a trusted third-party, does not involve in the authentication and key agreement process. It is only involved in the process of allocating long-term secret keys and their update. A lot of authentication protocols have been suggested for such situations. This article demonstrated that three state-of-the-art related protocols failed to remain anonymous, insecure against key compromise impersonation (KCI) attack, and clogging attack. To counter the pitfalls of them, a new D2D mutual authentication and key agreement protocol is designed here. The proposed protocol is anonymous, untraceable, and highly secure. Moreover, there is no need for a secure channel to generate a pair of private and public keys in the registration phase.) Formal security proof and security analysis using BAN logic, RealOr-Random (ROR) model, and Scyther tool showed that our proposed protocol satisfied security requirements. Furthermore, communication cost, computation cost, and energy consumption comparisons denoted our schema has better performance, compared to other protocols.
\end{abstract}

Keywords: Internet of Things (IoT), Device to Device (D2D) authentication, Key agreement, Key Compromise Impersonation (KCI) attack.

\section{1. introduction}

The promising prospect of $5 \mathrm{G}$ cellular networks and future-generation $6 \mathrm{G}$ networks and their wide applications in different areas make it hard to design them. Millions of people and devices are linked to each other with the Internet of Everything (IoE), often through wireless networks [1]. Therefore, they face many security risks. Different architectures have been proposed for IoT and IoE networks which consider communication infrastructures, communication range, computational power, and transmission capacity of IoT/IoE devices. Some include smart transportation systems, smart healthcare systems, internet of vehicles (IoV), electric vehicular network, distributed heterogeneous environment without surveillance, industrial applications, smart satellite communications, and UAV control [2].

Device-to-device (D2D) and machine-to-machine (M2M) communications have attracted great scholarly attention in this regard. Due to the lack of an authentication server as a trusted third party, this communication faces many challenges $[3,4]$. In mutual key-agreement protocols, a third-party authentication server is used in registration and initialization phases to load credentials, long keys, and other secret parameters. Two IoT devices can identify each other and share a secure session key without a trusted authority (TA). However, data transmitted in the public channels can be eavesdropped on by an adversary who performs some attacks such as violation of confidentiality, location forgery attack, user/devise impersonation, node compromise attack, and geographical position revealing. It can also breach anonymity and untraceability [5].

Alzahrani et al. [6] proposed an ECC-based key-agreement protocol for D2D communications in IoV networks that does not need a TA in the authentication and key-agreement phase. They analyzed and revealed security pitfalls of protocols by Islam and Biswas [7] and Mandal et al. [8]. In this article, we demonstrate that the protocol proposed by Alzahrani et al. [6] suffers from some problems in design and is insecure against insider attacks, key compromise attack, and fails to provide anonymity. On the other hand, the proposed protocol by 
Li et al. [9] for D2D-based communication is elaborated here and is shown to be vulnerable against key compromise attack and replay attack. Furthermore, it does not support anonymity and untraceability. Also, Chaudhry et al.' protocol [10] cannot counter the clogging attack. Thus, a novel protocol for D2D communications without the presence of a trusted third party to improve protocols by Alzahrani et al. [6], Chaudhry et al. [10], and Li et al. [9] is proposed here. The proposed protocol is highly efficient and secure. Also, it does not need a private channel in the registration phase for generating private-public keys. It also provides key update capability.

\subsection{Motivation}

Secure and effective interaction in Device-to-Device (D2D) and Machine Type Communication (MTC), without involving a trusted authority, is essential for $5 \mathrm{G}, 6 \mathrm{G}$, and its application. Exclusion of a trusted authority from authentication and key-agreement process may decrease delay and overhead, but may also bring about security challenges and impaired resistance against known attacks. This article offers a mutual authentication protocol between two IoT devices that are anonymous and untraceable while being effective and secure, making it a proper model for resource-restrained IoT devices. Here, IoT devices communicate with the trusted server only when they register or update the password in the network. Moreover, the private key is calculated by the IoT device and a single point failure problem is resolved.

\subsection{Contribution}

We analyzed protocols by Alzarani et al. [6], Chaudhry et al. [10], and Li et al. [9] in terms of security issues. Then, we proposed a mutual key-agreement protocol that includes four phases: (i) initialization, (ii) registration and long secret key generation, (iii) authentication and key agreement, (iv) public and private keys updating. The contributions of this article are listed below:

I) A two-party authenticated key agreement protocol is proposed to solve the key escrow problem without the involvement of an online Trusted Third-Party (TTP) authentication server.

II) Mutual authentication phase is done without a TA, and registration and the key updating phase are done in an insecure channel.

III) Three State-of-the-art related works and their security flaws are investigated.

IV) Strict formal security verification is achieved using the Scyther tool and BAN logic in the proposed protocol.

V) Resistance to different kinds of attacks is elaborated with the help of informal security verification.

VI) Evaluation of network performance, including communication/computation overheads and energy consumption, demonstrates that our proposed protocol is more effective compare to other protocols.

\subsection{Organization}

The rest of the article is organized as follows: Section 2 provides a review of the literature. Section 3 discusses the shortcomings of protocols proposed by Alzahrani et al., Chaudhry et al., and Li et al. Our proposed protocol is presented in section 4 . The security and efficiency of our protocol are evaluated in section 5. Finally, the conclusion and future work are given in section 6.

\section{Literature review}

Amin et al. [11] proposed a mutual key-agreement protocol between users and the cloud server for distributed and mobile cloud environments. They used bilinear pairing in the authentication phase which is not suitable for D2D communications because of excessive overhead. A lightweight authentication protocol based on hash and XOR operations for wearable devices was introduced by Das et al. [12]. But the protocol is not secure against desynchronization and offline guessing attacks. In [13], a protocol was designed for IoT environments that supports anonymity but allows forged nodes to penetrate into the network. Wu et al. [14] offered a mutual key agreement protocol for smart power grids to be applied on smart meter and smart service provider, but it is insecure against known session-specific temporary information attack. D2D communications are significant 
used in vehicular ad-hoc networks, especially vehicle-to-vehicle (V2V) communication. Li et al. [9] presented an identity-based V2V key agreement scheme. But it is not anonymous and provides no subtle mechanism to identify the vehicles. A mutual design was offered for body sensor networks by Shuai et al. [15]. A similar V2V protocol for IoT-based industrial environments was proposed by Lara et al. [16]. Islam [17] introduced a twofactor lattice-based encryption protocol for post-quantum cryptography to solve discrete logarithm problems and factoring problems in quantum environments. In order to reduce evaluation overhead and authenticate public keys, and to solve the key escrow problem, Islam and Biswas [7] designed an ECC-based key agreement protocol along with the self-certified public key for two-party communications. Mandal et al. [8] improved the security issues of Islam and Biswas' protocol. Nevertheless, Alzahrani et al. [6] showed that both protocols by Islam-Biswas [7] and Mandal et al. [8] suffered from key compromise impersonation attacks and do not ensure the anonymity of IoT devices. Chaudhry et al. [10] improved Das et al.'s [18] protocol (LACKA-IoT) and proposed iLACKA-IoT to access IoT-based cloud systems for D2D communication. They showed that LACKAIoT was insecure to man-in-the-middle attack and device impersonation attack. Table 1 has provided explanations for previous methods and features.

Table 1. Summary of the related works

\begin{tabular}{|c|c|c|c|}
\hline protocol & year & Features and good points & Weak points \\
\hline $\begin{array}{l}\text { Alzahrani } \\
\text { et al. [6] }\end{array}$ & 2020 & $\begin{array}{l}\text { ECC-based, D2D communication, review previous } \\
\text { work, self-certificate, security analysis using BAN } \\
\text { logic and random oracle model (ROM) }\end{array}$ & $\begin{array}{l}\text { Lack of anonymity } \\
\text { Vulnerable to key compromise impersonation } \\
\text { (KCI) attack }\end{array}$ \\
\hline $\begin{array}{l}\text { Islam and } \\
\text { Biswas [7] }\end{array}$ & 2015 & $\begin{array}{l}\text { ECC-based, D2D communication, review previous } \\
\text { work, self-certified public keys, security analysis } \\
\text { using AVISPA tool and ROM }\end{array}$ & $\begin{array}{l}\text { Lack of anonymity and mutual authentication, } \\
\text { vulnerable to KCI, replay, and clogging attacks }\end{array}$ \\
\hline $\begin{array}{l}\text { Mandal et } \\
\text { al. [8] }\end{array}$ & 2018 & $\begin{array}{l}\text { ECC-based, user-to-user interaction, review pervious } \\
\text { work, self-certificate, security analysis using AVISPA } \\
\text { tool and BAN logic }\end{array}$ & $\begin{array}{l}\text { Lack of anonymity } \\
\text { Vulnerable to KCI attack }\end{array}$ \\
\hline Li et al. [9] & 2019 & $\begin{array}{l}\text { ECC-based, vehicular ad hoc networks (VANET), } \\
\text { vehicle-to-vehicle (V2V), security analysis using } \\
\text { random oracle model }\end{array}$ & $\begin{array}{l}\text { Lack of anonymity and mutual authentication, } \\
\text { vulnerable to replay, clogging, and KCI attacks }\end{array}$ \\
\hline $\begin{array}{l}\text { Chaudhry } \\
\text { et al. [10] }\end{array}$ & 2020 & $\begin{array}{l}\text { ECC-based, IoT based sensor cloud systems, D2D, } \\
\text { review pervious work, ROR model }\end{array}$ & $\begin{array}{l}\text { Lack of anonymity and forward secrecy, } \\
\text { vulnerable to clogging attack }\end{array}$ \\
\hline $\begin{array}{l}\text { Amin et al. } \\
{[11]}\end{array}$ & 2016 & $\begin{array}{l}\text { bilinear pairing, user-to-cloud communication, } \\
\text { review pervious work, password renewal phase, } \\
\text { security analysis using AVISPA tool }\end{array}$ & $\begin{array}{l}\text { High computation and storage cost, poor } \\
\text { scalability, vulnerable to offline guessing attack } \\
\text { and insider attack }\end{array}$ \\
\hline $\begin{array}{l}\text { Das et al. } \\
{[12]}\end{array}$ & 2017 & $\begin{array}{l}\text { lightweight cryptography, wearable devices, user-to- } \\
\text { wearable device interaction, dynamic wearable } \\
\text { device addition, security analysis using real-or- } \\
\text { random (ROR) model and AVISPA tool }\end{array}$ & $\begin{array}{l}\text { Lack of forward secrecy } \\
\text { Vulnerable to desynchronization attack, and } \\
\text { offline guessing attack, }\end{array}$ \\
\hline $\begin{array}{l}\text { Simplicio } \\
\text { Jr et al. } \\
{[13]}\end{array}$ & 2016 & $\begin{array}{l}\text { ECC-based, review pervious work, combination of } \\
\text { strengthened-Menezes-Qu-Vanstone (SMQV) with } \\
\text { implicit certificates }\end{array}$ & $\begin{array}{l}\text { Lack of anonymity and formal security analysis, } \\
\text { vulnerable to KCI, impersonation, and replay } \\
\text { attacks }\end{array}$ \\
\hline $\begin{array}{l}\text { Wu et al. } \\
{[14]}\end{array}$ & 2019 & $\begin{array}{l}\text { ECC-based, smart grid architecture, ECC, Random } \\
\text { Oracle, ProVerif tool, Simulation, smart meter-to- } \\
\text { service provider }\end{array}$ & $\begin{array}{l}\text { Vulnerable to KCI attack, replay attack, and insider } \\
\text { attack }\end{array}$ \\
\hline $\begin{array}{l}\text { Shuai et al. } \\
\text { [15] }\end{array}$ & 2020 & $\begin{array}{l}\text { ECC-based, wireless body area networks, client-to- } \\
\text { application provider interaction, random oracle } \\
\text { model }\end{array}$ & Vulnerable to KCI attack \\
\hline $\begin{array}{l}\text { Lara et al. } \\
{[16]}\end{array}$ & 2020 & $\begin{array}{l}\text { Lightweight operation, M2M communications } \\
\text { (sensor-gateway), industrial internet of things } \\
\text { (IIoT), security analysis using AVISPA and BAN logic }\end{array}$ & $\begin{array}{l}\text { Vulnerable to desynchronization attack and secret } \\
\text { leakage attack }\end{array}$ \\
\hline $\begin{array}{l}\text { Das et al.'s } \\
{[18]}\end{array}$ & 2019 & $\begin{array}{l}\text { ECC-based, smart device (D2D), device access } \\
\text { method, ROR model, AVISPA tool, simulation }\end{array}$ & $\begin{array}{l}\text { Lack of anonymity, vulnerable to impersonation } \\
\text { attack and man-in-the-middle (MITM) attack }\end{array}$ \\
\hline
\end{tabular}


In this article, we analyze the protocols by Alzahrani et al. [6], Li et al. [9], and Chaudhry et al. [10] to demonstrate their security deficiencies. Our proposed protocol features an acceptable computational and communication overhead which, considering the real-time nature of D2D systems and resource scarcity, greatly improves performance. Table 2 summarizes symbols and corresponding definitions used in this article.

Table 2. Symbols and their definitions

\begin{tabular}{|c|l|c|l|}
\hline Symbol & Description & Symbol & Description \\
\hline$T A$ & Trusted Authority & $K_{p u b}, K_{p r i}$ & Public and Private key pair of $T A$ \\
\hline$D_{i}$ & $i^{\text {th } \text { IoT Device }}$ & $d_{i}, Q_{i}$ & Public and Private key pair of $D_{i}$ \\
\hline$I D_{i}$ & Identity of $D_{i}$ & $x, y$ & Random nonce chosen by $D_{i}$ and $D_{j}$ \\
\hline$T I D_{i}$ & Temporal identity computed by $D_{i}$ & $t_{X}$ & Current timestamps $(X=1,2)$ \\
\hline$p$ & A large prime number & $\mathcal{A}$ & Adversary \\
\hline$F_{p}$ & A prime finite field & $S K_{i j}$ & Session Key between $D_{i}$ and $D_{j}$ \\
\hline$Z_{p}^{*}$ & A set of prime numbers $\{1, \ldots, p-1\}(p>3)$ & $\Delta T$ & Maximum allowable transmission delay \\
\hline$G$ & Base point over $E / F_{p}$ & $\oplus, \|$ & Bitwise XOR, Concatenation operation \\
\hline & $\begin{array}{l}\text { An elliptic curve over prime field } F_{p} \text { defined by } \\
\text { non-singular elliptic curve equation: } \\
y^{2}=x^{3}+a x+b(\bmod p)\end{array}$ & $\begin{array}{l}\text { One-way and collision-resistant } \\
\text { cryptographic hash function defined } \\
h\{0,1\}^{*} \rightarrow h\{0,1\}^{l} \text { with arbitrary length inputs } \\
\text { and fixed length outputs. }\end{array}$ \\
\hline $\begin{array}{l}E(a, b) \\
/ F_{p}\end{array}$ & $\begin{array}{l}a, b \text { are constants. } x, y, a, b \in F_{p} \text { and } 4 a^{2}+ \\
27 b^{2}(\bmod p) \neq 0 .\end{array}$ & $h(\cdot)$ & \\
\hline
\end{tabular}

\section{Detailed review}

In this section, protocols proposed by Alzahrani et al. [6], Chaudhry et al [10], and Li et al [9] are discussed in detail in terms of security against known attacks.

\subsection{Threat model}

In our model, IoT devices communicate through a public channel. The following security issues are concerned with the adversary:

I) The adversary $\mathcal{A}$ cannot distinguish $x G$ and $x y G$.

II) The adversary is unlikely to retrieve $x$ from $h(x)$.

III) The adversary can eavesdrop, modify, remove, and duplicate messages transmitted in the public channel.

IV) The adversary can act as an insider to obtain secret parameters of other members in order to implement attacks.

V) The adversary can compromise all network entities and obtain all temporary as well as permanent credentials.

\subsection{Review of Alzahrani et al.'s protocol}

Alzahrani et al. [6] proposed an ECC-based key agreement protocol for IoT-based environments without TA intervention. The protocol included three phases: Initial system setup, registration, and authentication, which are described below.

3.1.1. Initial system setup phase. The server (a trusted third authority) calculates private key $K_{p r i}$ and public key $K_{p u b}=K_{p r i} G$ as $K_{p r i} \in Z_{p}^{*}$. It then publishes system parameters $\left\{E / F_{p}, h(), G,. K_{p u b}\right\}$ in the public space; where $G$ is the base point, $h($.$) is one-way hash function, and E / F_{p}$ is the Elliptical curve $E$ on finite field $F$ of order $q$. 
3.1.2. Registration phase. In this phase, device $D_{i}$ picks a unique identity $I D_{i}$ and random number $r_{i} \in Z_{p}^{*}$ to be registered on a network. Then, it computes $R_{i}=h\left(I D_{i} \| r_{i}\right) G$ and transmits the message $\left\langle I D_{i}, R_{i}\right\rangle$ to TA. Upon receiving the message, the trusted authority selects a random nonce $r_{i}^{T A} \in Z_{p}^{*}$ and calculates $R_{i}^{T A}=$ $h\left(I D_{i} \| r_{i}^{T A}\right) K_{\text {pub }}+R_{i}, f_{i}=\left[h\left(I D_{i} \| r_{i}^{T A}\right)+h\left(I D_{i} \| R_{i}^{T A}\right)\right] K_{\text {pri }}$, and $Q_{i}=R_{i}^{T A}+H\left(I D_{i} \| R_{i}^{T A}\right) K_{\text {pub }}$. After that, it sends $<I D_{i}, R_{i}^{T A}$, $f_{i}>$ via a secure channel to $D_{i}$, and publishes $Q_{i}$ as the public key of $D_{i}$. Finally, $D_{i}$ receives the second message and calculates private key $d_{i}$ as $d_{i}=\left[f_{i}+h\left(I D_{i} \| r_{i}\right)\right]$. If $Q_{i}=$ ? $d_{i} G=R_{i}^{T A}+h\left(I D_{i} \| R_{i}\right) G$ is met, it stores pair of $\left(d_{i}, Q_{i}\right)$ in its memory.

3.2.3. Authentication and key agreement phase. This phase is established between $D_{i}$ and $D_{j}$ and shown in Fig. 1.

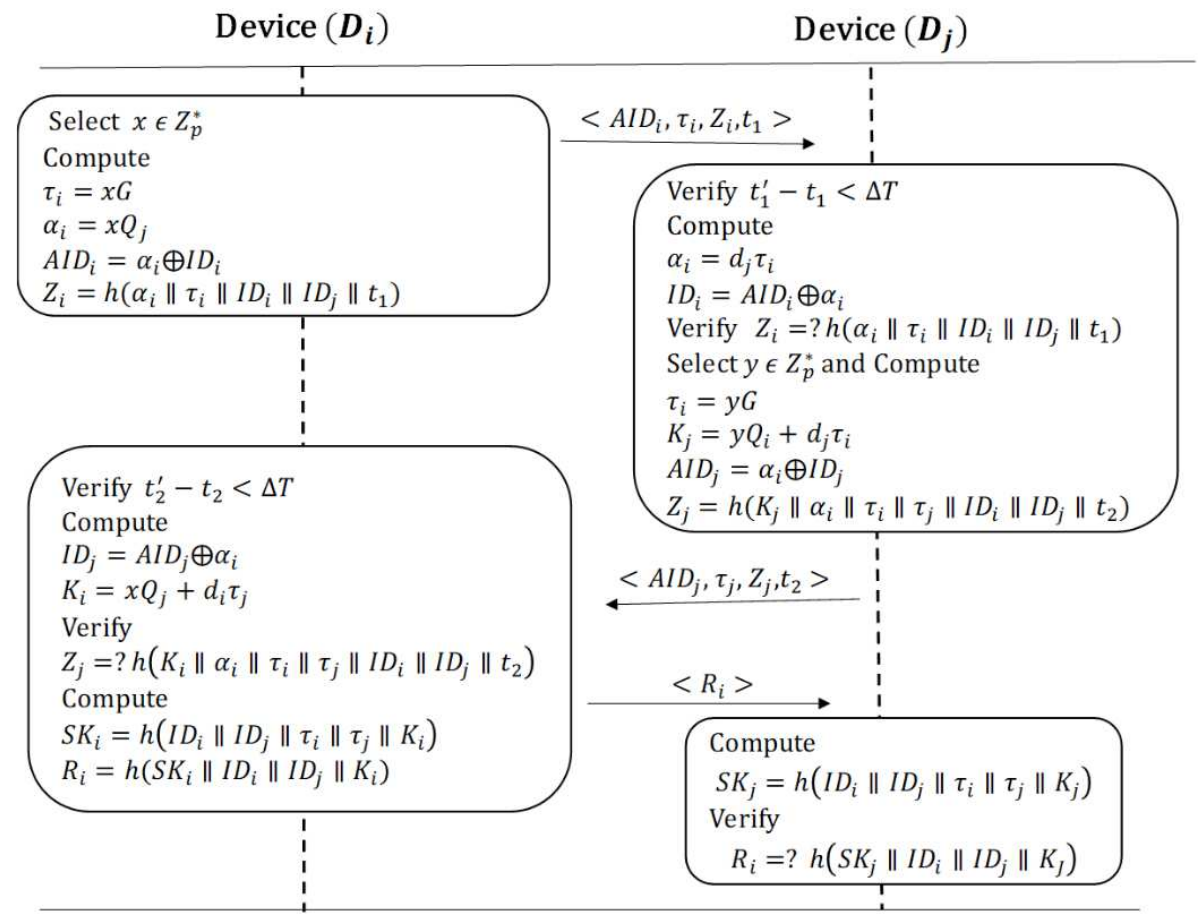

Fig. 1. Authentication process in Alzahrani et al.'s protocol

Step 1. $D_{i}$ chooses a random number $x \in Z_{p}^{*}$ and calculates values of $\tau_{i}=x G$ and $\alpha_{i}=x Q_{j}$ where $Q_{j}$ is the public key of $D_{j}$. Then, it calculates pseudo-ID $A I D_{i}=\alpha_{i} \oplus I D_{i}$ and calculates $Z_{i}$ as $Z_{i}=h\left(\alpha_{i}\left\|\tau_{i}\right\| I D_{i}\left\|I D_{j}\right\| t_{1}\right)$ where $t_{1}$ is the current timestamp of the message. Finally, the message $<A I D_{i}, \tau_{i}, Z_{i}, t_{1}>$ is sent to $D_{j}$.

Step 2. $D_{j}$ receives the message $<A I D_{i}, \tau_{i}, Z_{i}, t_{1}>$ at time $t_{1}^{\prime}$. If $t_{1}^{\prime}-t_{1}<\Delta T$, it verifies the freshness of the message. Then, it calculates $\alpha_{i}=d_{j} \tau_{i}$ and retrieves $I D_{i}=A I D_{i} \oplus \alpha_{i}$. After that, it checks whether or not $Z_{i}=$ $? h\left(\alpha_{i}\left\|\tau_{i}\right\| I D_{i}\left\|I D_{j}\right\| t_{1}\right)$ and verifies $D_{i}$. Otherwise, it immediately aborts the session. $D_{j}$ then, picks the random nonce $y \in Z_{p}^{*}$ and timestamp $t_{2}$ and calculates the values of $\tau_{i}=y G, K_{j}=y Q_{i}+d_{j} \tau_{i}, A I D_{j}=\alpha_{i} \oplus I D_{j}$, and $Z_{j}=h\left(k_{j}\left\|\alpha_{i}\right\| \tau_{i}\left\|\tau_{j}\right\| I D_{i}\left\|I D_{j}\right\| t_{2}\right)$. Finally, it transmits the message $<A I D_{j}, \tau_{j}, Z_{j}, t_{2}>$ to $D_{i}$.

Step 3. $D_{i}$ checks freshness of the received message and retrieves $I D_{j}=A I D_{j} \oplus \alpha_{i}$. Then, it calculates $K_{i}=x Q_{j}+$ $d_{i} \tau_{j}$ and checks if $Z_{j}=? h\left(k_{i}\left\|\alpha_{i}\right\| \tau_{i}\left\|\tau_{j}\right\| I D_{i}\left\|I D_{j}\right\| t_{2}\right)$. If the condition holds, $D_{j}$ is verified. $D_{i}$ then calculates the session key $S K_{i}$ as $S K_{i}=h\left(I D_{i}\left\|I D_{j}\right\| \tau_{i}\left\|\tau_{j}\right\| K_{i}\right)$ and generates message $<R_{i}>$ as $R_{i}=$ $h\left(S K_{i}\left\|I D_{i}\right\| I D_{j} \| K_{i}\right)$ to transmit to $D_{j}$. 
Step 4. Upon the reception of the message, $D_{j}$ calculates session key as $S K_{j}=h\left(I D_{i}\left\|I D_{j}\right\| \tau_{i}\left\|\tau_{j}\right\| K_{j}\right)$ and checks whether or not $R_{i}=? h\left(S K_{j}\left\|I D_{i}\right\| I D_{j} \| K_{j}\right)$. If not, it immediately terminates the session; otherwise, $S K=S K_{i}=S K_{j}$ is verified.

\subsection{Weakness of Alzahrani et al.'s protocol}

In this section, we will discuss the weaknesses in Alzahrani et al.'s protocol.

3.2.1. Problem 1. In the authentication phase, $A I D_{i}$ is calculated as $A I D_{i}=\alpha_{i} \oplus I D_{i}$, where $\alpha_{i}=x Q_{j}, Q_{j}$ is the public key of $D_{j}, x$ is a point on elliptical curve, and $I D_{i}$ is the unique identity of $D_{j}$. In practice, XOR operation may not be applied on them. Thus, $A I D_{i}=h\left(\alpha_{i}\right) \oplus I D_{i}$ is suggested instead.

3.2.2. Problem 2. $D_{i}$ has the value of $I D_{j}$ while generating $Z_{i}=h\left(\alpha_{i}\left\|\tau_{i}\right\| I D_{i}\left\|I D_{j}\right\| t_{1}\right)$. This decreases protocol scalability. Moreover, saving all IDs increases the risk of node compromise attack because once the adversary seizes a node's ID, he can fetch other IDs and violate protocol anonymity. On the other hand, because of node mobility, it is not efficient to stores all IDs in the memory.

3.2.3. Insecurity against privileged insider attack. An insider can get the ID of a device and reach the ID of all other connected devices, as explained below.

Step 1. The insider agent picks the random nonce $x^{*} \in Z_{p}^{*}$ and calculates values of $\tau_{i}^{*}=x^{*} G, \alpha^{*}=x^{*} Q_{j}$, and $A I D_{i}^{*}=\alpha_{i}^{*}+I D_{i}^{*}$. Then, it generates $Z_{i}^{*}=h\left(\alpha_{i}^{*}\left\|\tau_{i}^{*}\right\| I D_{i}^{*}\left\|I D_{j}\right\| t_{1}\right)$ and transmits the message $<A I D_{i}^{*}, Z_{i}^{*}$, $\tau_{i}^{*}, t_{1}>$ to $D_{j}$.

Step 2. $D_{j}$ checks for verifies the freshness of the message and calculates $A I D_{j}=\alpha_{i}^{*} \oplus I D_{j}$, and sends it to $D_{i}^{*}$.

Step 3. Upon the reception of $A I D_{j}, D_{i}^{*}$ obtains $I D_{j}=\alpha_{i}^{*} \oplus A I D_{j}$. $D_{i}^{*}$ eavesdrops all transmissions from $D_{j}$ to other devices and obtains $A I D_{j}^{\text {new }}=\alpha_{j}^{\text {new }} \oplus I D_{j}$. Applying XOR operation, it achieves $\alpha_{j}^{\text {new }}$. Hence, protocol secrecy is violated.

Step 4. Once the insider obtains $\alpha_{j}^{\text {new }}$, he applies XOR operation on $A I D_{j}^{\text {new }} \oplus \alpha_{j}^{\text {new }}$ to obtain $I D_{j}^{\text {new }}$ of the device. Thus, Alzahrani et al.'s protocol loses anonymity and untraceability. To solve this problem, a temporary ID is suggested to be used for communications.

3.2.4. Key Compromise Impersonation (KCI) attack. Once a key is compromised by the adversary, the replay attack could run as follows.

Step 1. Similar to the insider attack, the adversary $\mathcal{A}$ obtains the key $d_{i}$ and IDs of nodes. $\mathcal{A}$ then selects $x^{A} \in Z_{p}^{*}$ and calculates $\tau_{i}^{A}=x^{A} G, \alpha_{i}^{A}=x^{A} Q_{i}, A I D_{i}^{A}=\alpha_{i}^{A} \oplus I D_{i}, Z_{i}^{A}=h\left(\alpha_{i}^{A}\left\|\tau_{i}^{A}\right\| I D_{i}\left\|I D_{j}\right\| t_{1}\right)$, and transmits the message $<A I D_{i}^{A}, \tau_{i}^{A}, Z_{i}^{A}, t_{i}>$ to $D_{j}$.

Step 2. $D_{j}$ receives the first message $A I D_{i}^{A} \oplus \alpha_{i}^{A}$ and obtains $I D_{i}$. It checks if $Z_{i}^{A}=$ ? $h\left(\alpha_{i}^{A}\left\|Z_{i}^{A}\right\| I D_{i}\left\|I D_{j}\right\| t_{1}\right)$ and authenticates $I D_{i}$. Thus, the adversary $\mathcal{A}$ can easily forge a device. Hence, their protocol is vulnerable against key compromise impersonation attack.

\subsection{Review of Li et al.'s protocol}

The proposed protocol by Li et al. includes three phases as described below.

3.3.1 Initial setting phase. TA creates a finite cyclic additive group $\mathbb{G}$ with generator $G$ and chooses a big prime number $p$. Then, it selects the private key $K_{p r i} \in Z_{p}^{*}$ and calculates the public key $K_{p u b}=K_{p r i} G$, and chooses hash functions $h_{1}(),. h_{2}(),. h_{3}($.$) and publishes parameters <\mathbb{G}, p, G, K_{p u b}, h_{1}(),. h_{2}(),. h_{3}()>$.. 
3.3.2. Registration phase. The vehicle $V_{i}$ selects an $I D_{i}$ and sends it to TA through a secure channel. TA chooses a random number $r_{i} \in Z_{p}^{*}$ and calculates $R_{i}=r_{i} G$. It then calculates $h_{i}=h_{1}\left(I D_{i} \| R_{i}\right)$ and $d_{i}=r_{i}+$ $h_{i} K_{P r i}(\bmod p)$ and transmits the pair $\left(d_{i}, R_{i}\right)$ to $V_{i}$ through a secure channel. $V_{i}$ checks whether or not $d_{i} G=$ $? R_{i}+h_{1}\left(I D_{i} \| R_{i}\right) K_{p u b}$. If the condition holds, it accepts $Q_{i}=d_{i} G$ as its public key.

3.3.3 Key agreement phase. This phase occurs between the vehicles $V_{i}$ and $V_{j}$ without an intervention of a thirdparty server. This phase is demonstrated in Fig. 2.

Step 1. $V_{i}$ selects a random number $x \in Z_{p}^{*}$ and calculates $\tau_{i}=x G$, and sends $\left\{I D_{i}, R_{i}, \tau_{i}\right\}$ to $V_{j}$.

Step 2. Upon reception of the message, $V_{j}$ selects a random number $y \in Z_{p}^{*}$ and $\tau_{i}=y G$ and sends the message $<I D_{j}, R_{j}, \tau_{j}>$ to $V_{i}$. At the same time, it obtains the public key of $V_{i}$ as $Q_{i}=R_{i}+h_{1}\left(I D_{i} \| R_{i}\right) K_{p u b}$ and calculates $K_{j}=h_{2}\left(d_{j} Q_{i}\right) y \tau_{i}$ and the session key $S K_{j}=h_{3}\left(I D_{i}\left\|I D_{j}\right\| K_{j}\left\|y Q_{i}\right\| d_{j} \tau_{i}\right)$.

Step 3. $V_{i}$ receives the message $<I D_{j}, R_{j}, \tau_{j}>$ and obtains public key $Q_{j}=R_{j}+h_{1}\left(I D_{j} \| R_{j}\right) K_{p u b}$, and calculates $K_{i}=h_{2}\left(d_{i} Q_{j}\right) x \tau_{j}$ and obtains the session key $S K_{i}=h_{3}\left(I D_{i}\left\|I D_{j}\right\| K_{i}\left\|x Q_{j}\right\| d_{i} \tau_{j}\right)$.

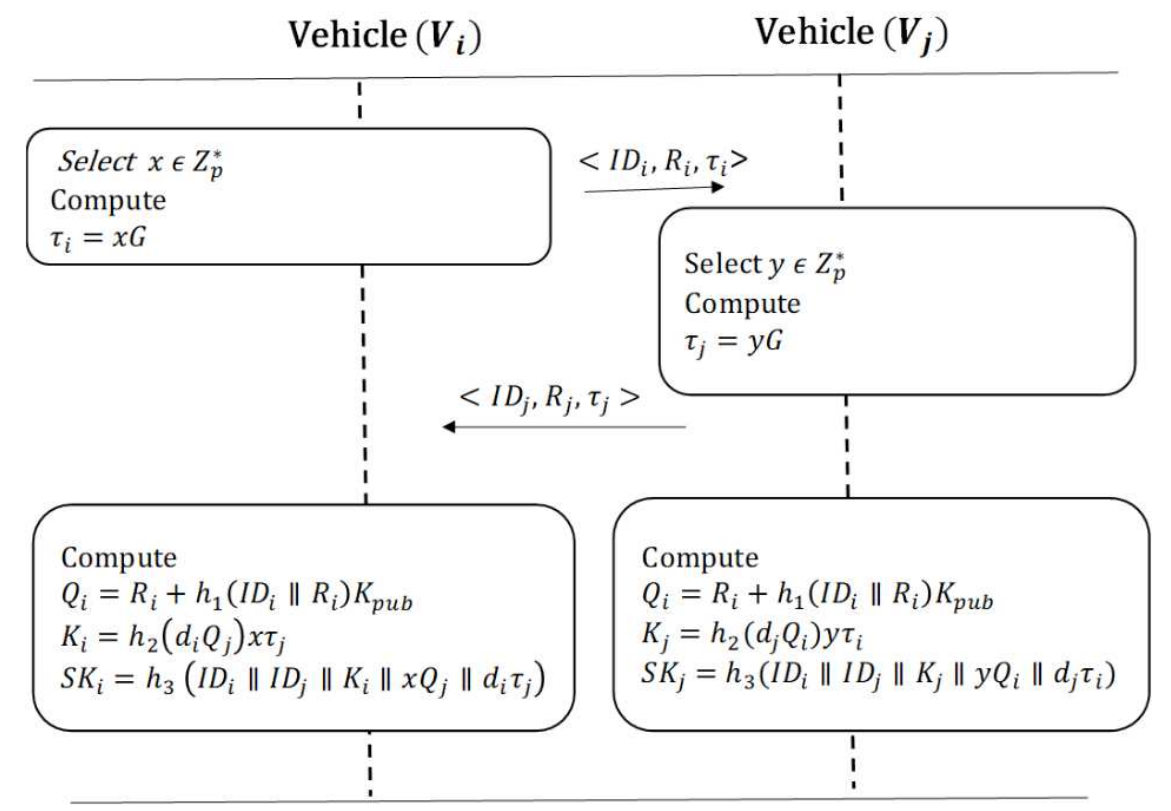

Fig. 2. Authentication process in Li et al.'s protocol

\subsection{Weakness of Li et al.'s protocol}

The following summarizes security issues of Li et al.'s protocol.

3.4.1. Lack of mutual authentication. $V_{i}$ and $V_{j}$ focus on session key agreement and fail to authenticate each other. Thus, the adversary $\mathcal{A}$ can easily insert forged messages into the network.

3.4.2. Clogging attack. It is a subclass of DoS attacks wherein the adversary clogs the receiver and wastes its communication and computation sources in an attempt to paralyze the receiver [8]. In Li et al.'s protocol, the adversary runs the clogging attack as follows:

Step 1. Adversary $\mathcal{A}$ captures the first message $\left\langle I D_{i}, R_{i}, \tau_{i}>\right.$ in key agreement phase. $\mathcal{A}$ then selects a random nonce $x^{A} \in Z_{p}^{*}$ and calculates $\tau^{A}=x^{A} G$, and transmits the message $\left\langle I D_{i}, R_{i}, \tau^{A}>\right.$ to $V_{j}$. 
Step 2. Upon receiving the message, $V_{j}$ selects a random nonce $y$ and calculates $\tau_{j}=y G$, public key $Q_{i}=R_{i}+$ $h_{1}\left(I D_{i} \| R_{i}\right) K_{p u b}$, and session key $S K_{j}^{A}=h_{3}\left(I D_{i}\left\|I D_{j}\right\| K_{j}\left\|y Q_{i}\right\| d_{j} \tau^{A}\right)$. Then, it transmits the message $<I D_{j}$, $R_{j}, \tau_{j}>$ to $\mathcal{A}$.

Step 3. Adversary $\mathcal{A}$ transmits the message $<I D_{j}, R_{j}, \tau^{A}>$ to $V_{i}$ with only calculating $\tau^{A}=x G$.

Step 4. $V_{i}$ receives the message and calculates $Q_{j}=R_{j}+h_{1}\left(I D_{j} \| R_{j}\right) K_{p u b}, K_{i}=h_{2}\left(d_{i} Q_{j}\right) x \tau^{A}$, and session key $S K_{i}^{A}=h_{3}\left(I D_{i}\left\|I D_{j}\right\| K_{i}\left\|x Q_{j}\right\| d_{i} \tau^{A}\right)$.

This attack desynchronizes the agreed session key between the agents, i.e., $S K_{j}^{A} \neq S K_{i}^{A}$. Adversary $\mathcal{A}$ performs a multiplication operation of ECC and makes the two agents $V_{i}$ and $V_{j}$ run 11 scalar multiplication, 2 point addition, and 6 hash function. This causes a huge loss of time and costs that is only recognized by desynchronized session key after transmission of encrypted data.

3.4.3. Lack of anonymity and untraceability. In Li et al.'s protocol, vehicle IDs are transmitted clearly and an adversary can easily track and store messages to use them for its own purposes. Hence, their protocol loses anonymity and untraceability.

3.4.4. Replay attack. Transmitted messages include $x$ and $y$ nonces but have no timestamp. Because of traffic constraints of vehicles and IoT devices, they cannot store all the nonces. Thus, an adversary can capture the first $<I D_{i}, R_{i}, \tau_{i}>$ or the second $\left\langle I D_{j}, R_{j}, \tau_{j}>\right.$ message and transmit it later to be verified by the receiver. It is, therefore, vulnerable to replay attack.

3.4.5. Key compromise impersonation. If private keys $d_{i}$ and $d_{j}$ are revealed, the adversary can easily forge a vehicle as follows:

Step 1. The adversary $\mathcal{A}$ obtains $d_{i}, I D_{i}$ and $R_{i}$ and uses them to obtain public key $Q_{i}$ as $Q_{i}=R_{i}+h_{1}\left(I D_{i} \|\right.$ $\left.R_{i}\right) K_{\text {pub }}$. Then, $\mathcal{A}$ selects a random nonce $x^{A} \in Z_{p}^{*}$ and calculates $\tau^{A}=x^{A} G$, and transmits the message $<I D_{i}, R_{i}, \tau^{A}>$ to $V_{j}$.

Step 2. Upon receiving the message, $V_{j}$ retrieves the public key $Q_{i}$ because there are no changes in $I D_{i}$ and $R_{i}$. Then, session keys $S K_{i}$ and $S K_{j}$ will be equal as follows: $S K_{i}^{A}=h_{3}\left(I D_{i}\left\|I D_{j}\right\| h_{2}\left(d_{j} Q_{i}\right) x^{A} \tau_{j}\left\|x^{A} Q_{j}\right\| d_{i} \tau_{j}\right)=$ $h_{3}\left(I D_{i}\left\|I D_{j}\right\| h_{2}\left(d_{i} Q_{j}\right) y \tau^{A}\left\|y Q_{i}\right\| d_{j} \tau^{A}\right)=S K_{j}^{A}$. Therefore, once $d_{i}$ is revelaed, the adversary can forge a node.

\subsection{Analysis of Chaudhry et al.'s protocol}

In Chaudhry et al.'s protocol, a TA selects $I D_{i}$ and private key $d_{i} \in Z_{p}^{*}$ and uses random nonce $l_{i} \in Z_{p}^{*}$ to calculate the certificate $C_{i}$ of private key $Q_{i}$, as $Q_{i}=d_{i} G, A_{i}=\left(d_{i}+l_{i}\right) G, C_{i}=K_{p r i}\left(d_{i}+l_{i}\right) h\left(I D_{i} \| A_{i}\right)+d_{i}$ where $K_{p r i}$ is private key of TA. These are used in D2D authentication and key agreement between two IoT devices. An authentication and key agreement phase in this protocol is shown in Figure 3. 


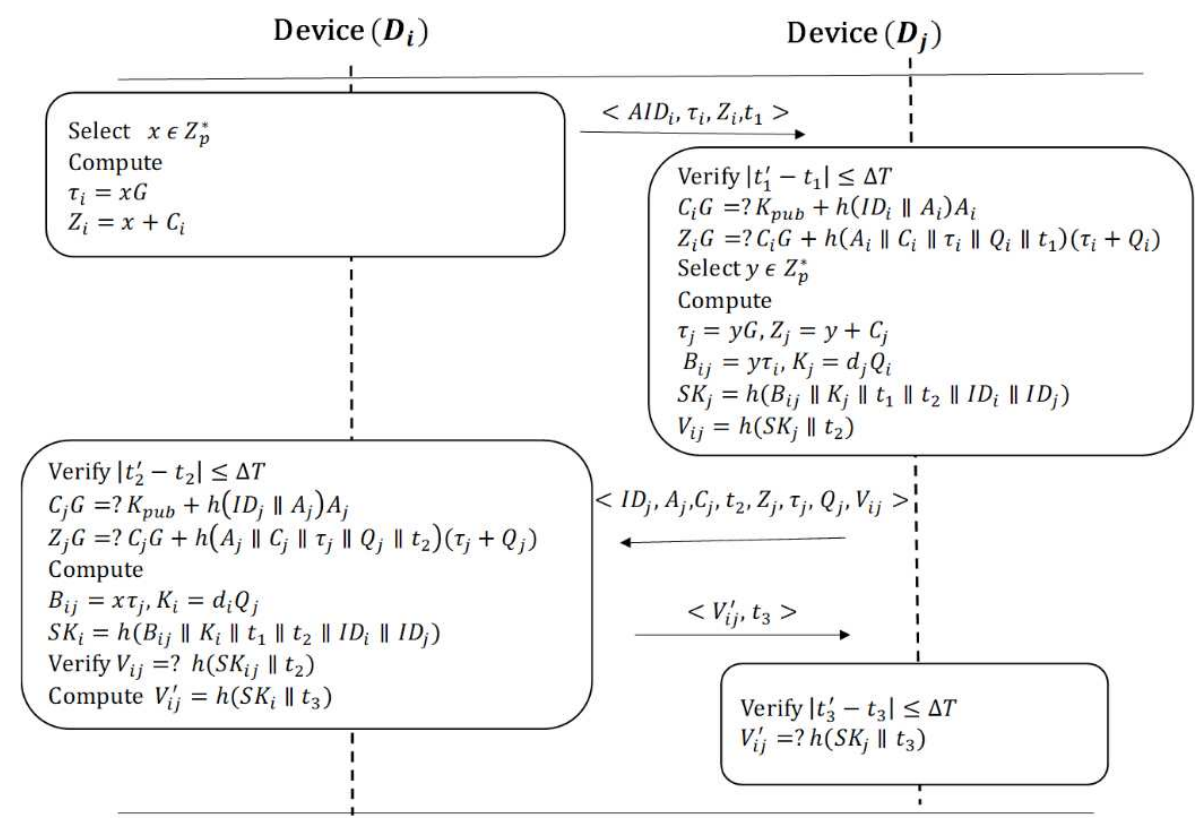

Fig. 3. Authentication process in Chaudhry et al.'s protocol

3.5.1. Lack of anonymity and untraceability. Since $I D_{i}$ and $I D_{j}$ for $D_{i}$ and $D_{j}$ devices are clearly transferred in the channel, the adversary can easily eavesdrop messages. Therefore, anonymity and untraceability are breached in this protocol.

3.5.2. Clogging Attack. Chaudhry et al.'s protocol is insecure against clogging attack as shown below.

Step 1 . The adversary $\mathcal{A}$ captures the message $\left\langle I D_{i}, A_{i}, t_{1}, Z_{i}, \tau_{i}, Q_{i}>\right.$ that consists the current timestamp $t_{1}$, $\tau_{i}=x G$, and $Z_{i}=x+C_{i}$, is transmitted by $D_{i}$ in which $x \in Z_{p}^{*}$. Then, $\mathcal{A}$ generates random nonce $x^{A}$ and calculates $\tau_{i}^{A}=x^{A} G, \tau_{i}^{A^{\prime \prime}}=\tau_{i}^{A}+\tau_{i}$, and $Z_{i}^{A}=Z_{i}+x^{A}=\left(x+C_{i}+x^{A}\right)$. After that, the adversary transmits the fake message $<I D_{i}, A_{i}, t_{1}, Z_{i}^{A}, \tau_{i}^{A^{\prime \prime}}, Q_{i}>$ to $D_{j}$.

Step 2. Upon reception of the message, $D_{j}$ checks its freshness $Z_{i}^{A} G ?=\tau_{i}^{A^{\prime \prime}}+K_{p u b}+h\left(I D_{i} \| A_{i}\right) A_{i}+Q_{i}$ to ensure:

$Z_{i}^{A} G=\left(x+C_{i}+x^{A}\right) G=x^{A} G+x G+C_{i} G=x^{A} G+x G+K_{p r i} G+h\left(I D_{i} \| A_{i}\right)\left(d_{i}+l_{i}\right) G+Q_{i}=\tau_{i}^{A}+\tau_{i}+$ $K_{\text {pub }}+h\left(I D_{i} \| A_{i}\right)\left(d_{i}+l_{i}\right) G+Q_{i}=\tau_{i}^{A^{\prime \prime}}+K_{\text {pub }}+h\left(I D_{i} \| A_{i}\right) A_{i}+Q_{i}$

The condition is met and the adversary can deceive $D_{j}$ and breach message integration. This waste $D_{j}$ resources because it will perform 4 ECC multiplication operations and 2 hash functions to transmit the message $<I D_{j}, A_{j}$, $t_{2}, Z_{j}, \tau_{j}, Q_{j}, V_{i j}>$ to $D_{i}$. Then, $D_{i}$ will do some more calculations and realize that the session key is different, implying that clogging attack has been successful.

3.5.3. Lack of perfect forward secrecy. Once the adversary compromises $d_{i}, C_{i}$ of $D_{i}$, $\mathrm{s} /$ he obtains agreed session keys in authentication phase. This is because $C_{i}$ is fixed and retrievable in mutual authentication between $D_{i}$ and $D_{j}$. The following elaborates on this attack:

Step 1. $\mathcal{A}$ eavesdrops and saves the messages $<I D_{i}, A_{i}, t_{1}, Z_{i}, \tau_{i}, Q_{i}>$ and $<I D_{j}, A_{j}, t_{2}, Z_{j}, \tau_{j}, Q_{j}, V_{i j}>$ from earlier sessions. 
Step 2. $\mathcal{A}$ captures $D_{i}$ to obtain long-term credentials $d_{i}$ and $C_{i}=K_{p r i}\left(d_{i}+l_{i}\right) h\left(I D_{i} \| A_{i}\right)+d_{i}$. These two parameters are fixed. The adversary needs to obtain $B_{i j}=y \tau_{i}=x \tau_{j}$ and $K=d_{i} Q_{j}=d_{j} Q_{i}$ to retrieve session keys from earlier sessions as $S K_{i j}^{\text {old }}=h\left(B_{i j}^{\text {old }}\|K\| t_{1}\left\|t_{2}\right\| I D_{i} \| I D_{j}\right)$.

Step 3. Adversary $\mathcal{A}$ uses $Z_{i}^{\text {old }}$ to obtain $x^{\text {old }}$, and then calculates $K=d_{i} Q_{j}, x^{\text {old }}=Z_{i}^{\text {old }}-C_{i}$, and $B_{i j}{ }^{\text {old }}=$ $x^{\text {old }} \tau_{j}^{\text {old }}$. Thus, the session key from the earlier session is recalled. In the same way, the keys of the future sessions can be obtained. Hence, Chaudhry et al.'s protocol fails to support perfect forward/backward secrecy.

\section{System description and proposed protocol 4.1 Network model}

6G technology is not limited to cellular mobile networks and is aimed at expanding digital communications. However, improve effectiveness in the real-time communication and dense IoT networks requires reducing computational and communication overhead. However, adversaries and insiders can penetrate into the network due to the use of insecure channels and temporary connections for high network mobility. Thus, in addition to promoting performance, security, and privacy issues need to be considered.

The proposed model includes three major entities: IoT devices, Trusted authority (TA), and an adversary. IoT devices are $6 \mathrm{G}$ communication devices that communicate with their peers or far servers with no human involvement. In fact, they provide Machine Type Communication (MTC).

TA provides offline information for IoT devices. Considering scalability and widespread use of $6 \mathrm{G}$ networks, it is more optimal for devices to register online in their preferred network. These IoT devices include sensors embedded on smart vehicles, sensors in smart houses, smart health systems, UAVs, smart agriculture, and other single-hop or hierarchical networks. Fig 4. demonstrates the network model.

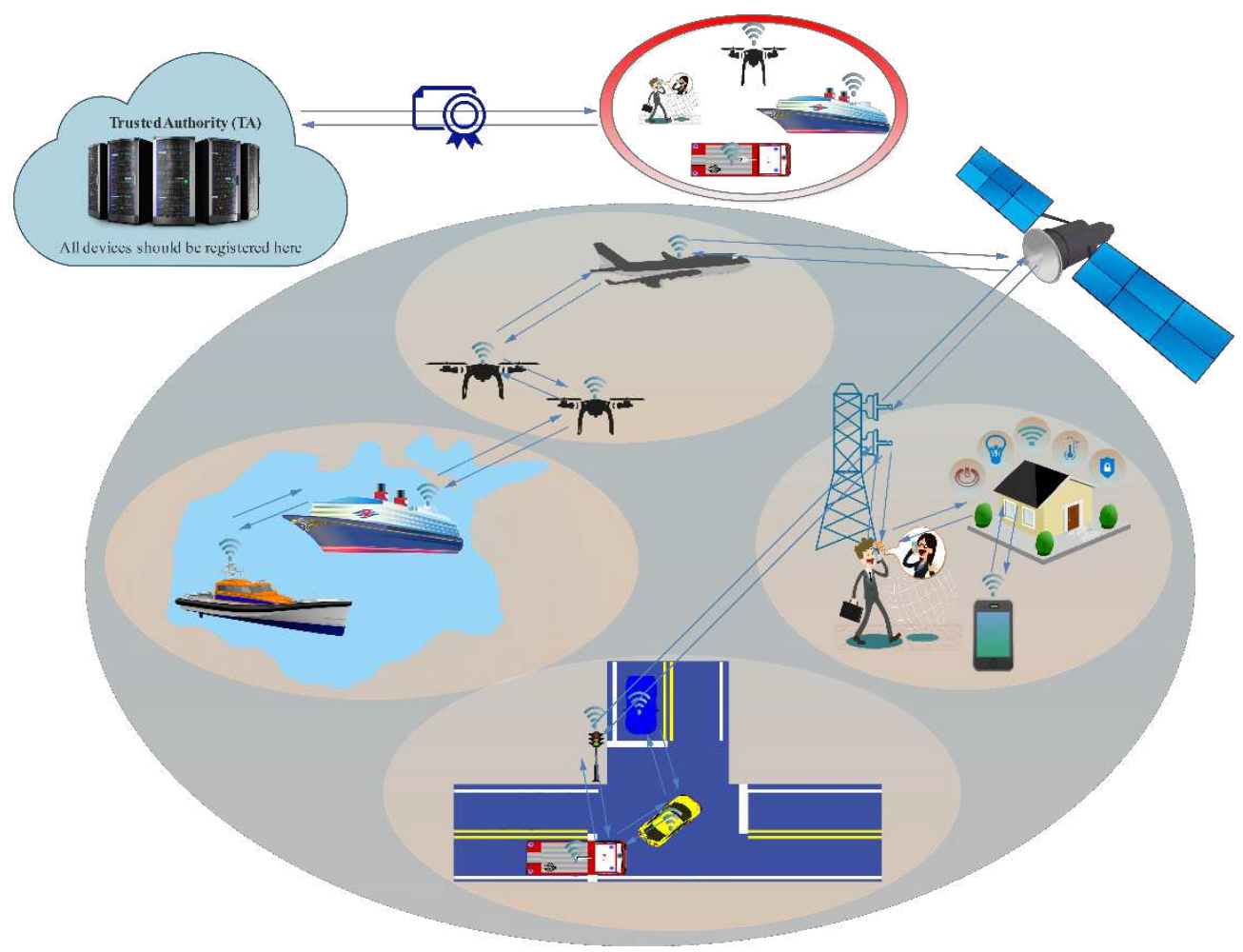

Fig. 4. Network model 


\subsection{Communication model}

An IoT device may communicate with another device or a TA through channels including Bluetooth, Wi-Fi, ZigBee, cellular spectrum, optical fiber, etc. We analyze the most challenging communication scenario between two devices and TA-free authentication.

\subsection{Proposed protocol}

In this section, a mutual key agreement and authentication protocol for IoT devices without the intervention of a Trusted Authority (TA) is proposed. It includes four phases: initial system configuration, registration and key generation, authentication and key agreement, public and private keys updating. TA intervenes in initialization, the long-secret key generating, and updating phases to allocate device-specific public keys. To avoid key escrow problem, in case the TA's key is disclosed, the private keys of devices are not exposed. This is because each device separately calculates its private key. The proposed protocol uses ECC encryption, one-way hash function, and XOR operation. ECC-based encryption is advantageous for its high security compared to symmetric encryption and short key length compared to asymmetric encryption such as RSA. This increases computational performance and security features. All the phases in the protocol, except the first phase, are done in a public channel that is accessible to the invader. A summary of the first and second phases of the proposed protocol is demonstrated in Figure 5.

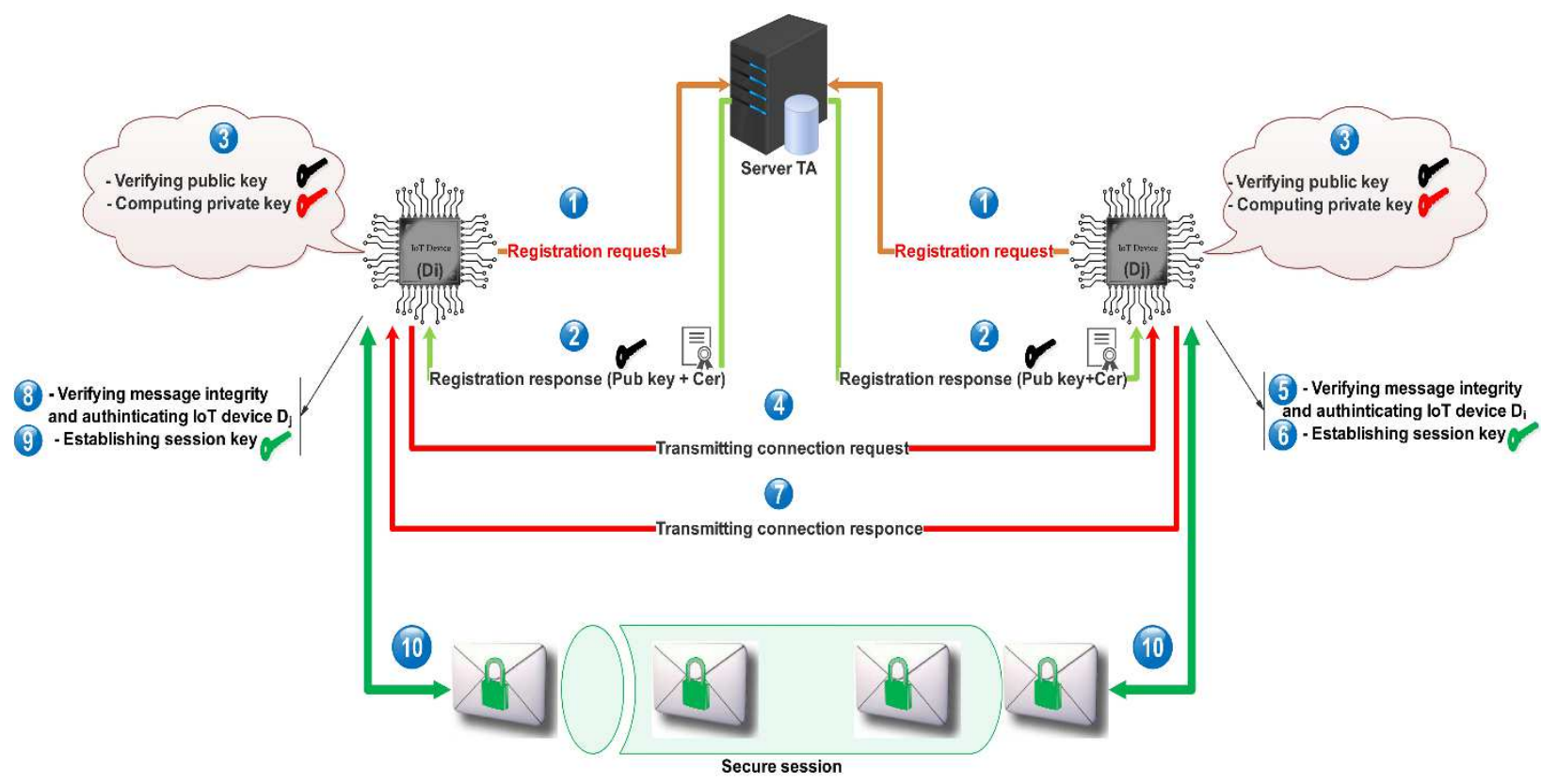

Fig. 5. An overview of the second and third phases of the proposed protocol

\subsubsection{Initial system configuration}

In this phase, TA selects Elliptic curve $E$ on finite field $F$ with big prime order $p$ and collision resistant one-way hash function $h($.$) . TA, then selects the private key K_{p r i} \in Z_{p}^{*}$ for itself and calculates the corresponding public key $K_{p u b}=K_{p r i} G$. It also selects random nonce $s \in Z_{p}^{*}$ and assigns unique IDs to each IoT devices and stores $H I D_{x}=h\left(I D_{x}\|s\| K_{p r i}\right), s G$ in them. Finally, TA publishes public system parameters including $\left.\left\{E / F_{p}, h(), G,. K_{\text {pub }}\right\}.\right)$

\subsubsection{IoT device registration and key generation}

This phase occurs between IoT device $D_{i}$ and trusted server TA in a public channel as follows:

Step RKG1. $D_{i}$ selects the random nonce $r_{i} \in Z_{p}^{*}$ and unique $I D_{i}$. 
It then calculates $R_{i}=r_{i} G$, masked identity $M I D_{i}=I D_{i} \oplus h\left(r_{i} s G\right)$, and message authentication code $M A C_{i}=$ $h\left(I D_{i}\left\|H I D_{i}\right\| t_{1}\right)$. After that, it transmits message $<M I D_{i}, R_{i}, M A C_{i}, t_{1}>$ in timestamp $t_{1}$, to TA through a public channel.

Step RKG2. TA receives the message $<M I D_{i}, R_{i}, M A C_{i}, t_{1}>$, and retrieves $I D_{i}=M I D_{i} \oplus h\left(s R_{i} G\right)$. It then checks the freshness of the message and computes $H I D_{i}=h\left(I D_{i}\|s\| K_{\text {pri }}\right)$ and verifies message integrity by checking $M A C_{i}=$ ? $h\left(I D_{i}\left\|H I D_{i}\right\| t_{1}\right)$. If the condition is met, it selects a random nonce $e_{i} \in Z_{p}^{*}$ and obtains values of $P_{i}=$ $e_{i} K_{p u b}+R_{i}, f_{i}=\left(e_{i}+P_{i}\right) K_{\text {pri }}$ and public key $Q_{i}=P_{i}+P_{i} K_{\text {pub }}$. Also, TA calculates $M_{i}=K_{p r i} Q_{i}$ to obtain values of $N_{i}=M_{i}+h\left(I D_{i}\right) G, F_{i}=f_{i} \oplus h\left(I D_{i} \| R_{i}\right)$ and $M A C_{T A}=h\left(H I D_{i}\left\|N_{i}\right\| F_{i}\right)$. Finally, the second message < $F_{i}, N_{i}, M A C_{T A}>$ is sent to $D_{i}$ through a public channel. TA calculates $\xi_{i}=e_{i} \oplus h\left(R_{i} \| K_{p r i}\right)$ and stores $\xi_{i}$ and $R_{i}$ in its memory. It publishes $P_{i}$ and public key $Q_{i}$ in the public space.

Step RKG3. Upon receiving the message $\left\langle F_{i}, N_{i}, M A C_{T A}>, D_{i}\right.$ retrieves $f_{i}=F_{i} \oplus h\left(I D_{i} \| R_{i}\right)$ and calculates its private key $d_{i}$ as $d_{i}=f_{i}+r_{i}$, then verifies $Q_{i}=$ ? $d_{i} G$ to ensure accuracy of its calculations. After that, it retrieves $M_{i}=N_{i}-h\left(I D_{i}\right) G$ and Checks the validity of $M A C_{T A}=$ ? $h\left(H I D_{i}\left\|N_{i}\right\| F_{i}\right)$. I=If the equality test is satisfied, $d_{i}$ and $Q_{i}$ are considered to be legal pairs of private and public keys. The public key $Q_{i}$ and $d_{i} G$ are equal, as below.

$d_{i} G=\left(f_{i}+r_{i}\right) G=\left(\left[\left(e_{i}+P_{i}\right) K_{p r i}\right] G+R_{i}=e_{i} K_{p u b}+R_{i}+P_{i} K_{p u b}=P_{i}+P_{i} K_{p u b}=Q_{i}\right.$

Thus, TA calculates and sends $Q_{i}$ to the IoT device $D_{i}$ without having access to private key $d_{i}$. This phase is briefly demonstrated in Fig 6.

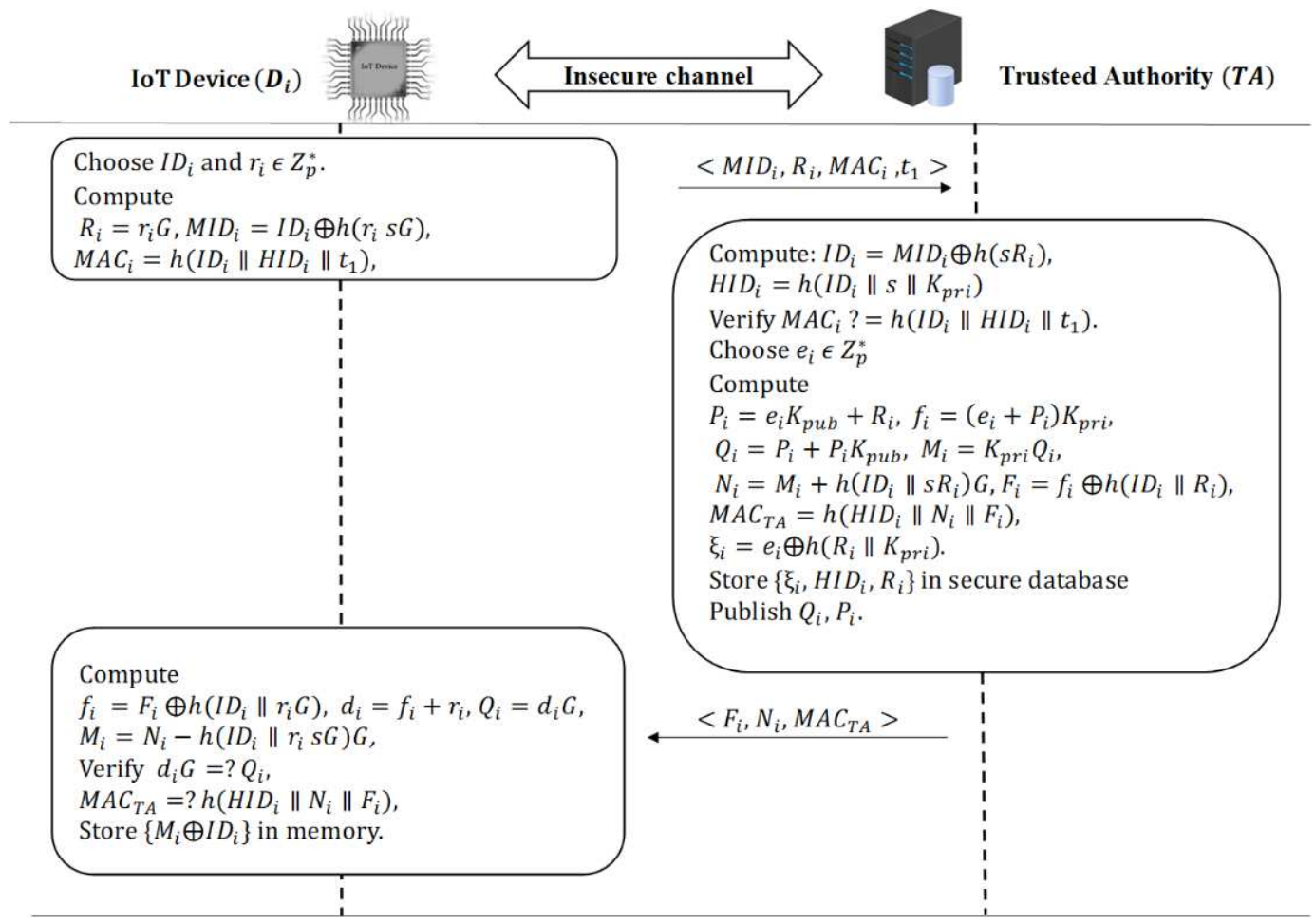

Fig. 6. IoT Device registration and key generation phase

\subsubsection{Authentication and key agreement phase}

Two IoT devices $D_{i}$ and $D_{j}$ need to authenticate each other and agree on a shared session key to establish a secure connection. Then, they encrypt their information by the session key $S K_{i j}$ and publish that in the public channel. Authentication and key agreement are done in the public channel where adversaries may be present. This phase is described below in three steps. 


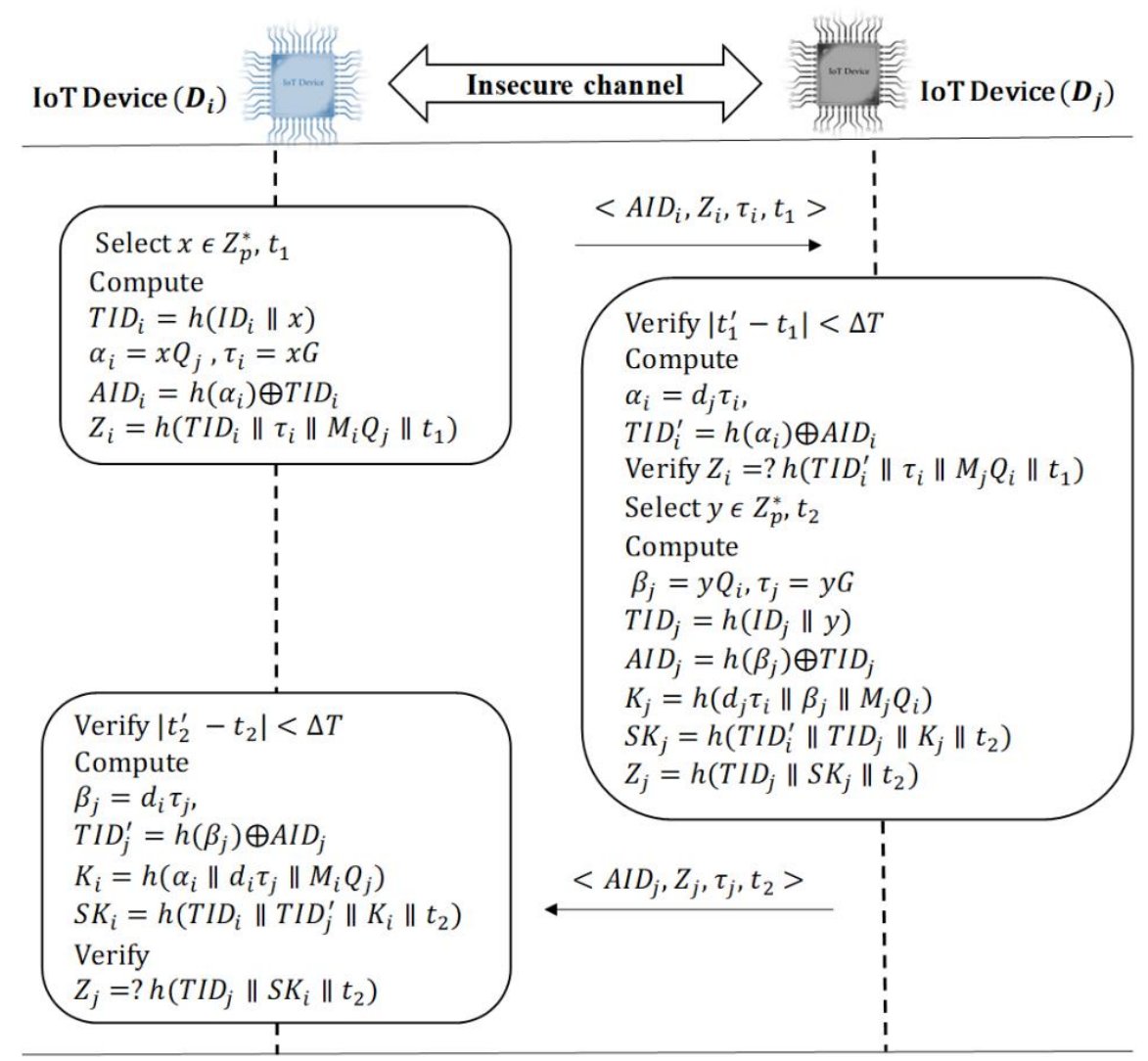

Fig. 7. Authentication and key agreement phase

Step AKA1: $D_{i} \rightarrow D_{j}:<A I D_{i}, Z_{i}, \tau_{i}, t_{1}>$

The IoT device $D_{i}$ selects a temporary identity $T I D_{i}$, a random nonce $x \in Z_{p}^{*}$, and current timestamp $t_{1}$. It then calculates $\alpha_{i}=x Q_{j}, \tau_{i}=x G, A I D_{i}=h\left(\alpha_{i}\right) \oplus T I D_{i}$, and $Z_{i}=h\left(T I D_{i}\left\|\tau_{i}\right\| M_{i} Q_{j} \| t_{1}\right)$. Finally, it transmits the message $<A I D_{i}, Z_{i}, \tau_{i}, t_{1}>$ to the IoT device $D_{j}$.

Step AKA2: $D_{j} \rightarrow D_{i}:<A I D_{j}, Z_{j}, \tau_{j}, t_{2}>$

Upon receiving the message $\left\langle A I D_{i}, Z_{i}, \tau_{i}, t_{1}\right\rangle, D_{j}$ checks its freshness by verifying the condition $\left|t_{1}^{\prime}-t_{1}\right|<$ $\Delta T$, where $t_{1}^{\prime}$ is the time of message delivery. If the condition is met, $D_{j}$ calculates $\alpha_{i}=d_{j} \tau_{i}$ and $\operatorname{TID}_{i}^{\prime}=$ $h\left(\alpha_{i}\right) \oplus A I D_{i}$, where $d_{j}$ is the private key of $D_{j}$. It then checks $Z_{i}=$ ? $h\left(T I D_{i}^{\prime}\left\|\tau_{i}\right\| M_{j} Q_{i} \| t_{1}\right)$ to verify the equality authentication test. If the condition is not met, it immediately aborts the session; otherwise, verifies the message and $T I D_{i}$ as a legal entity. $D_{j}$ selects $T I D_{j}$ and random nonce $y \in Z_{p}^{*}$, and calculates $\beta_{j}=y Q_{i}, \tau_{j}=y G$, and $A I D_{j}=h\left(\beta_{j}\right) \oplus T I D_{j}$. After that, it calculates $K_{j}=h\left(d_{j} \tau_{i}\left\|\beta_{j}\right\| M_{j} Q_{i}\right)$ using public key of $D_{i}$, the value of $M_{j}$ in its memory, secret nonce $y$, and $\tau_{i}$ of the first message. Next, it calculates temporary session key $S K_{j}=$ $h\left(T I D_{i}^{\prime}\left\|T I D_{j}\right\| K_{j} \| t_{2}\right)$ where $t_{2}$ is the current timestamp of message generated by $D_{j}$. It also calculates $Z_{j}=$ $h\left(T I D_{j}\left\|S K_{j}\right\| t_{2}\right)$ to preserve message integrity, and transmits the message $<A I D_{j}, Z_{j}, \tau_{j}, t_{2}>$ to $D_{i}$ through a public channel.

Step AKA3: $D_{i} \leftarrow<A I D_{j}, Z_{j}, \tau_{j}, t_{2}>$

$D_{i}$ receives the message $<A I D_{j}, Z_{j}, \tau_{j}, t_{2}>$ and verifies its freshness by checking $\left|t_{2}^{\prime}-t_{2}\right|<\Delta T$. It then retrieves $\beta_{j}=d_{i} \tau_{j}$ and $T I D_{j}^{\prime}$ as $T I D_{j}^{\prime}=h\left(\beta_{j}\right) \oplus A I D_{j}$. Next, $D_{i}$ calculates $K_{i}=h\left(\alpha_{i}\left\|d_{i} \tau_{j}\right\| M_{i} Q_{j}\right)$ using public key $Q_{j}$, stored value $M_{i}$, nonce $x$, and $\tau_{j}$. Then, it calculates $S K_{i}=h\left(T I D_{i}\left\|T I D_{j}^{\prime}\right\| K_{i} \| t_{2}\right)$ and $Z_{j}^{*}=h\left(T I D_{j} \|\right.$ 
$S K_{i} \| t_{2}$ ). $D_{i}$ checks whether or not $Z_{j}=$ ? $Z_{j}^{*}$. If the condition holds, it authenticates $D_{j}$, and verifies $S K_{i}=S K_{j}$ as a secure session key; otherwise, it immediately aborts the session. Since $M_{i}=K_{p r i} Q_{i}$ and $M_{j}=K_{p r i} Q_{j}$ are delivered to $D_{i}$ and $D_{j}$ in registration phase, $K_{i}$ and $K_{j}$ are equal as $K_{j}=h\left(d_{j} \tau_{i}\left\|\beta_{j}\right\| M_{j} Q_{i}\right)=h\left(d_{j} x G\left\|\beta_{j}\right\|\right.$ $\left.K_{p r i} Q_{j} Q_{i}\right)=h\left(x Q_{j}\left\|y Q_{i}\right\| K_{p r i} Q_{i} Q_{j}\right)=h\left(\alpha_{i}\left\|d_{i} y G\right\| M_{i} Q_{j}\right)=h\left(\alpha_{i}\left\|d_{i} \tau_{j}\right\| M_{i} Q_{j}\right)=K_{i}$. Eventually, $S K_{i}=$ $S K_{j}$. This phase is briefly demonstrated in Fig 7.

\subsubsection{Public and Private Keys updating phase}

An IoT device may have to update private and public keys because of key expiration or disclosure. In our proposed protocol, the pair of private and public keys are updated through a public channel between the IoT device and TA, as follows.

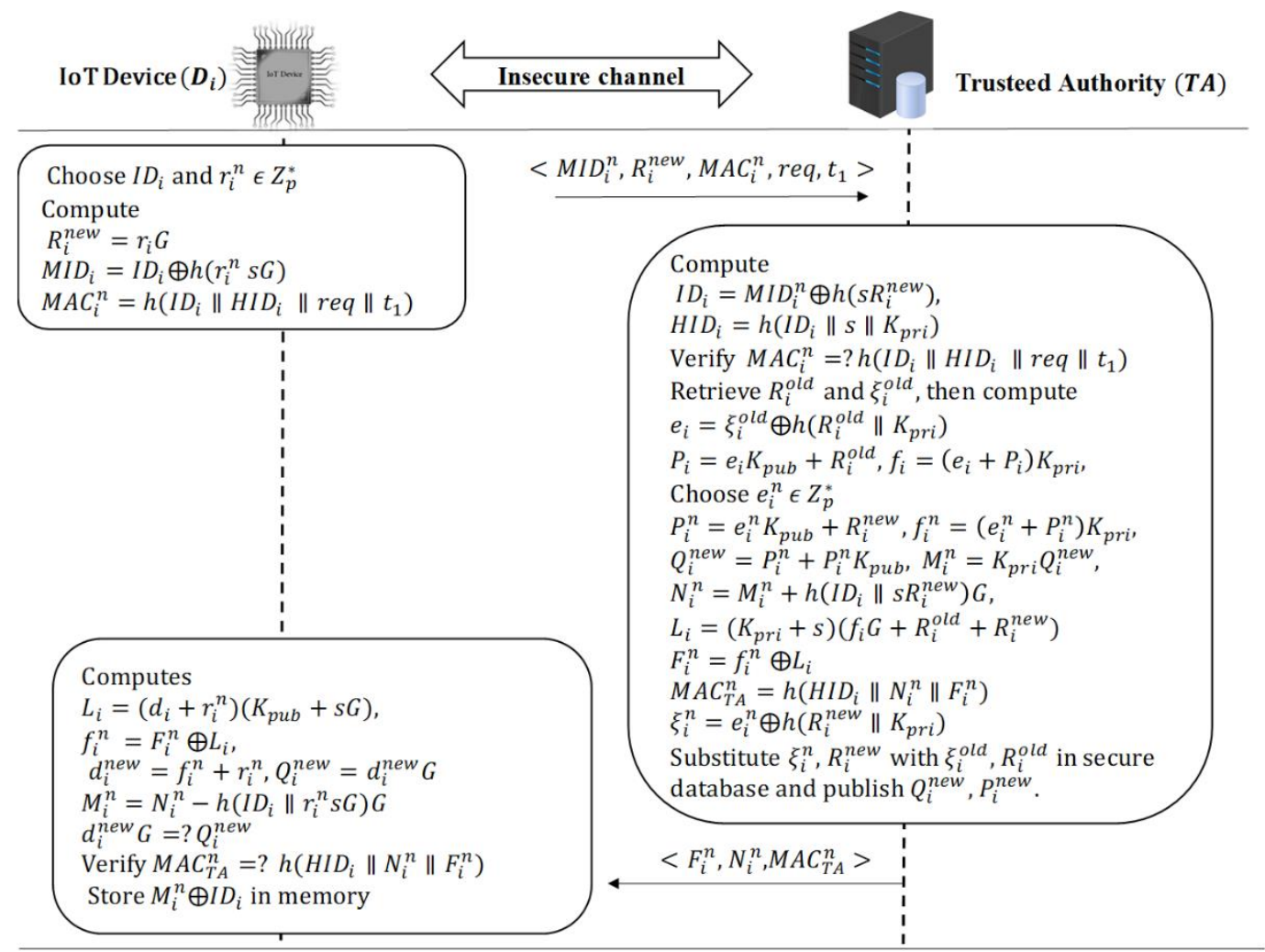

Fig .8. Updating pair of private and public keys phase

\section{Step KU1: $D_{i} \rightarrow T A:<M I D_{i}^{n}, R_{i}^{n e w}, M A C_{i}^{n}, r e q, t_{1}>$}

$D_{i}$ selects the random nonce $r_{i}^{n} \in Z_{p}^{*}$. It then calculates $R_{i}^{\text {new }}=r_{i}^{n} G$ and masked identity $M I D_{i}^{n}=I D_{i} \oplus h\left(r_{i}^{n} s G\right)$, $M A C_{i}^{n}=h\left(I D_{i}\left\|H I D_{i}\right\| r e q \| t_{1}\right)$ and transmits message $<M I D_{i}^{n}, R_{i}^{\text {new }}, M A C_{i}^{n}$, req, $t_{1}>$ to TA through a public channel. $t_{1}$ indicates the current timestamp of generating message.

\section{Step KU2: $T A \rightarrow D_{i}:<F_{i}^{n}, N_{i}^{n}, M A C_{T A}^{n}>$}

TA receives the message $\left\langle M I D_{i}^{n}, R_{i}^{\text {new }}, M A C_{i}^{n}\right.$, req, $\left.t_{1}\right\rangle$, and retrieves $I D_{i}=M I D_{i} \oplus h\left(s R_{i}^{\text {new }} G\right)$. It calculates $H I D_{i}=h\left(I D_{i}\|s\| K_{p r i}\right)$ and check the equality test $M A C_{i}^{n}=$ ? $h\left(I D_{i}\left\|H I D_{i}\right\| r e q \| t_{1}\right)$. If condition is not met, it terminate the session; otherwise, it recalls the corresponding values of $\xi_{i}^{\text {old }}$ and $R_{i}^{\text {old }}$ and computes $e_{i}=$ $\xi_{i}^{\text {old }} \oplus h\left(R_{i}^{\text {old }} \| K_{\text {pri }}\right), P_{i}=e_{i} K_{\text {pub }}+R_{i}^{\text {old }}$, and $f_{i}=\left(e_{i}+P_{i}\right) K_{\text {pri }}$. After that, it selects a random nonce $e_{i}^{n} \in Z_{p}^{*}$ and obtains values of $P_{i}^{n}=e_{i}^{n} K_{\text {pub }}+R_{i}^{\text {new }}, f_{i}^{n}=\left(e_{i}^{n}+P_{i}^{n}\right) K_{\text {pri }}$ and new public key $Q_{i}^{\text {new }}=P_{i}^{n}+P_{i}^{n} K_{\text {pub }}$. Also, 
TA calculates $M_{i}^{n}=K_{\text {pri }} Q_{i}^{\text {new }}$ to obtain values of $N_{i}^{n}=M_{i}^{n}+h\left(I D_{i} \| s R_{i}^{\text {new }}\right) G, F_{i}^{n}=f_{i}^{n} \oplus L_{i}$, and $L_{i}=\left(K_{\text {pri }}+\right.$ s) $\left(f_{i} G+R_{i}^{\text {old }}+R_{i}^{\text {new }}\right)$. Finally, it calculates $M A C_{T A}^{n}=h\left(H I D_{i}\left\|N_{i}^{n}\right\| F_{i}^{n}\right)$ and transmits the second message < $F_{i}^{n}, N_{i}^{n}, M A C_{T A}^{n}>$ to $D_{i}$ through a public channel. TA also calculates $\xi_{i}^{n}=e_{i}^{n} \oplus h\left(R_{i}^{\text {new }} \| K_{\text {pri }}\right)$ and replaces $\xi_{i}^{\text {old }}, R_{i}^{\text {old }}$ with $\xi_{i}^{n}, R_{i}^{\text {new }}$. It publishes new public key $Q_{i}^{\text {new }}$ and $P_{i}^{n}$ in the public space.

Step KU3: $D_{i} \leftarrow<F_{i}^{n}, N_{i}^{n}, M A C_{T A}^{n}>$

Upon receiving the message $\left\langle F_{i}^{n}, N_{i}^{n}, M A C_{T A}^{n}>, D_{i}\right.$ calculates $L_{i}=\left(d_{i}+r_{i}^{n}\right)\left(K_{p u b}+s G\right)$. Then, it retrieves $f_{i}^{n}=F_{i}^{n} \oplus L_{i}$ and calculates new public key $d_{i}^{\text {new }}$ as $d_{i}^{\text {new }}=f_{i}^{n}+r_{i}^{n}$ and verifies new public key $Q_{i}^{\text {new }}$ as $Q_{i}^{\text {new }}=? d_{i}^{\text {new }} G$. Then, it retrieves $M_{i}^{n}=N_{i}^{n}-h\left(I D_{i}\right) G$ and stores $M_{i}^{n}=N_{i}^{n}-h\left(I D_{i}\right) G$ in its memory. Two secrets $L_{i}=\left(d_{i}+r_{i}^{n}\right)\left(K_{\text {pub }}+s G\right)$ and $\left(K_{\text {pri }}+s\right)\left(f_{i} G+R_{i}^{\text {old }}+R_{i}^{\text {new }}\right)$ are equal as $L_{i}=\left(d_{i}+r_{i}^{n}\right)\left(K_{\text {pub }}+\right.$ $s G)=d_{i} K_{\text {pub }}+d_{i} s G+r_{i}^{n} K_{\text {pub }}+r_{i}^{n} s G=K_{\text {pri }}\left(f_{i}+r_{i}^{\text {old }}\right) G+K_{\text {pri }} r_{i}^{n} G+s\left(f_{i}+r_{i}^{\text {old }}\right) G+s r_{i}^{n} G=\left(K_{p r i}+\right.$ $s)\left(f_{i} G+R_{i}^{\text {old }}+R_{i}^{\text {new }}\right)$. Figure 8. demonstrate this phase.

\section{Security analysis and efficiency}

In this section, the proposed protocol is analyzed formally and informally to demonstrate the mutual authentication between two participants and shares a secure and temporary session key that is safe against well-known attacks.

\subsection{Security proof}

Real-or-Random (ROR) model $[10,18,19]$ is used to prove semantic security of the proposed protocol and to obtain session key security (SK-security), as shown in theorem 1.

\subsubsection{Preliminaries}

Only two partnerships (see definition 1) exist for session key establishing in the proposed protocol. TA actively engages in registration and public key assignment. Public parameters are $\left\{E / F_{p}, h(), G,. K_{p u b}\right\}$. Values of $\left\{M_{i}, I D_{i}, d_{i}, Q_{i}\right\}$ are stored in IoT device memory. An entity may have several instances known as oracles. TA server and the IoT device are two entities in the network. IoT devices include instances of $D e v^{i}$ and $D e v^{j}$. Oracles have three modes. (i) accept: the oracle receives a correct message and responds to it. It denotes a situation in which the last message by the oracle is accepted before the session expires. (ii) reject: the oracle receives a wrong message. (iii) null: the oracle returns no responses. $P I D^{i}$ and $P I D^{j}$ imply partnership identity of instances of $D e v^{i}$ and $D e v^{j} . S I D^{i j}$ is the current session ID. The following definitions help clarify our analyses.

Definition 1. (Participant). Includes all entities of IoT devices with their specific private and public keys in the proposed model.

Definition 2. (Partnering). Suppose $D e v^{i}$ and $D e v^{j}$ identified as $P I D^{i}$ and $P I D^{j}$ generate session $\mathcal{S}$ with the identity $S I D^{i j}$. If $D e v^{i}$ and $D e v^{j}$ have similar session IDs in accepted state that are directly linked to each other and complete a successful session, they will establish a partnership, one being the initiator and the other responder.

Definition 3. (Freshness). Session $\mathcal{S}$ is fresh if the adversary issues queries of SKReveal(Dev), EphReveal(Dev), Corrupt(Dev), and Compromise(Dev) while it or its matching session is at risk. These queries need to be requested before $S K^{i j}$ expires. This is to distinguish fresh and random session keys.

Definition 4. (Difference lemma). Suppose $S u c c_{n}^{\mathcal{A}} \mid n=1,2,3$ denote the event in probability density function and $\operatorname{Succ}_{1}^{\mathcal{A}} \wedge \neg \operatorname{Succ}_{3}^{\mathcal{A}} \Leftrightarrow \operatorname{Succ}_{2}^{\mathcal{A}} \wedge \neg \operatorname{Succ}_{3}^{\mathcal{A}}$. Then we have $\left|\operatorname{Pr}\left[\operatorname{Succ}_{1}^{\mathcal{A}}\right]-\operatorname{Pr}\left[\operatorname{Succ}_{2}^{\mathcal{A}}\right]\right| \leq \operatorname{Pr}\left[\operatorname{Succ}_{3}^{\mathcal{A}}\right]$.

The adversary can request the following queries to breach semantic security of the proposed protocol. 
$\boldsymbol{S e n d}\left(\boldsymbol{D e v}{ }^{i}, \boldsymbol{D e v}^{j},<\boldsymbol{m}_{\mathbf{1}}>\right)$. Once this query is implemented, $\mathcal{A}$ transmits the message $m_{1}=<A I D_{i}, Z_{i}, \tau_{i}, t_{1}>$ instead of $D e v^{i}$ to $D e v^{j} . D e v^{j}$ checks for validity of the query and, as specified above (see sec 4.2.3), calculates the session key $S K^{j}=h\left(T I D_{i}^{\prime}\left\|T I D_{j}\right\| K_{j} \| t_{2}\right)$ and returns the message $<A I D_{j}, Z_{j}, \tau_{j}, t_{2}>$.

$\boldsymbol{S e n d}\left(\boldsymbol{D e v}{ }^{j}, \boldsymbol{D e v}{ }^{i},<\boldsymbol{m}_{2}>\right)$. $\mathcal{A}$ sends the message $m_{2}=<A I D_{j}, Z_{j}, \tau_{j}, t_{2}>$ to $\operatorname{Dev}^{i}$ to forge the oracle $\operatorname{Dev}^{j}$. upon receiving the message, $D e v^{i}$ checks for values of the query and, as specified above, calculates the session key $S K^{i}=h\left(T I D_{i}\left\|T I D_{j}^{\prime}\right\| K_{i} \| t_{2}\right)$ and completes the session. If the conditions are not met or the session expires, the query is rejected.

Execute(Dev). For this query, $\mathcal{A}$ obtains $<m_{1}>$ and $<m_{2}>$ in authentication and key agreement phase. It is like a passive eavesdropping attack.

$\operatorname{Hash}(\operatorname{Var})$. The oracle Dev generates a list $L_{H}$ to store hash records. When the adversary issues Hash(Var), Dev searches in the list $L_{H}$ and returns the corresponding $v$ in tuple $(\operatorname{Var}, v)$. Otherwise, it generates a random value $v^{\prime}$ and adds $\left(\operatorname{Var}, v^{\prime}\right)$ to its list, returning the $v^{\prime}$.

SKReveal(Dev). It simulates session key leakage by the adversary. If $S K^{i j}$ is generated, Dev returns it in response to the query. Otherwise, it returns null.

EphReveal $\left(\boldsymbol{D e v}^{i}\right) . \mathcal{A}$ uses this query to obtain ephemeral secret parameters $\alpha$ and $x$ of $D e v^{i}$ to perform ephemeral secret leakage attack.

$\operatorname{Corrupt}\left(\boldsymbol{D e v}{ }^{i}\right)$. By running this query, the adversary obtains long-static secret parameters $d_{i}$ and $M_{i}$ of $D e v^{i}$.

Compromise $\left(\boldsymbol{D e v} v^{i}\right)$. By querying this, all static and dynamic secret parameters of $\operatorname{Dev}^{i}\left(x, \alpha_{i}, M_{i}\right.$ and $\left.d_{i}\right)$ are delivered to the adversary. The objective is to implement a successful insider attack to capture the whole network.

TestID(Dev). Once this is requested, Dev flips the coin $c=\{0,1\}$. If $c=1$, it returns the original ID to $\mathcal{A}$. Otherwise, it generates a string of random bits of similar length and returns in response.

TestSK(Dev). The adversary needs to have successfully implemented SKReveal(Dev) to run this query in an attempt to obtain the session key $S K^{i j}$ and disrupt semantic security. Upon receiving the query, Dev returns null if the key has not been generated. Otherwise, it flips a neutral coin. If adversary's guess $\left(c^{\prime}\right)$ and flipped coin $(c)$ are equal, it yields the session key to the adversary. Otherwise, it generates a random value of similar length and returns it in response.

Expire(Dev). It removes the session key generated by Dev.

Definition 5. (Semantic security). To simulate semantic security of the proposed protocol, we designed a series of consecutive and undistinguishable games between the adversary and oracle $D e v$. The adversary issues different queries to Dev to successfully lunch an attack. The adversary tries to guess the flipped coin $c$ through a TestSK query to increase his chance of winning. This query is asked when Dev is in accept state and the session $\mathcal{S}$ is fresh and not expired. Dev returns $S K$ when session key is generated and $c=c^{\prime}$.

The advantage of the adversary in breaching our protocol in the semantic security model is $A d v_{\mathcal{P}}^{\mathcal{A}}(t)=$ $\left|\operatorname{Pr}\left[\operatorname{Succ}_{\mathcal{P}}^{\mathcal{A}}(t)\right]-1\right|$, where $A d v_{\mathcal{P}}^{\mathcal{A}}(t) \leq \varepsilon$ and $\varepsilon>0$ is a trivial value.

\subsubsection{Theorem}

Suppose $\mathcal{A}$ a probabilistic polynomial adversary at time $t$ that seeks to breach semantic security of the proposed protocol. If $\mathcal{A}$ can issue maximum $q_{s}$ Send, $q_{h}$ Hash, and $q_{e}$ Execute queries, its advantage for winning the proposed protocol in consecutive games $G_{n} \mid n=1, \ldots, 8$ will be less than the following value because of the one-way hash function $h($.) and difficulty of ECCDHP. The output string of hash oracle is $\lambda$. And $\mathfrak{I}$ is the number of uncompromised instances of $D e v$ in the network. The adversary's likelihood to breach the semantic security of the proposed protocol is calculated as follows: 


$$
A d v_{\mathcal{P}}^{\mathcal{A}}(t) \leq \frac{5 q_{s}+4 q_{h}^{2}+\left(q_{s}+q_{e}\right)^{2}}{2 \lambda}+q_{h}^{2} A d v_{E C C D H P}(t)
$$

Eventually, the adversary's likelihood to compromise the whole network is calculated as follows:

$$
A d v_{N e t}^{\mathcal{A}}(t)=\prod_{i=1}^{\mathfrak{T}} A d v_{\mathcal{P}(i)}^{\mathcal{A}}(t)
$$

\subsubsection{Proof}

To prove the robustness of the proposed protocol, the following games $G_{n} \mid n=1, \ldots, 8$ are simulated between the adversary and $\operatorname{Dev}$. $\operatorname{Succ}_{n}^{\mathcal{A}}(t)$ is the chance of winning $n^{\text {th }}$ game by the adversary and $\operatorname{Pr}\left[\operatorname{Succ}_{n}^{\mathcal{A}}(t)\right]$ is the probability of winning at time $t$ in game $G_{n}$. Dev returns a response based on accept or reject state of the query. The games are simulated based on ROR as follows:

Game $\boldsymbol{G}_{\mathbf{1}}$. It simulates a real attack to the protocol. The adversary $\mathcal{A}$ needs to correctly guess the flipped coin. Thus, the probability of winning for $\mathcal{A}$ in real protocol with random oracles is

$$
A d v_{1}^{\mathcal{A}}(t)=2\left|\operatorname{Pr}\left[\operatorname{Succ}_{1}^{\mathcal{A}}(t)\right]-1\right|
$$

Game $\boldsymbol{G}_{\mathbf{2}}$. The adversary simulates Send and Execute queries in a real attack to obtain $\left\langle m_{1}\right\rangle$ and $\left\langle m_{2}\right\rangle$ messages, or tries to forge Dev. Thus, the probability of winning for the adversary is

$$
A d v_{1}^{\mathcal{A}}(t)=A d v_{2}^{\mathcal{A}}(t)
$$

Game $\boldsymbol{G}_{\mathbf{3}}$. It simulates a situation in which the adversary wins the game because of hash oracle collision. When $\mathcal{A}$ issues the query, $\mathrm{Dev}$ returns the appropriate response from $L_{T}$ and $L_{H}$ lists that store transcripts and hash records, respectively. The game is over when random numbers or hash oracles collide. Based on birthday paradox, the probability of collision for hash oracles and random numbers is $\frac{q_{h}^{2}}{2 \lambda}$ and $\frac{\left(q_{s}+q_{e}\right)^{2}}{2 \lambda}$, respectively. This game is undistinguishable from the previous one, and thus

$$
\left|\operatorname{Pr}\left[\operatorname{Succ}_{3}^{\mathcal{A}}(t)\right]-\operatorname{Pr}\left[\operatorname{Succ}_{2}^{\mathcal{A}}(t)\right]\right| \leq \frac{q_{h}^{2}+\left(q_{s}+q_{e}\right)^{2}}{2 \lambda}
$$

Game $\boldsymbol{G}_{\mathbf{4}}$. It simulates the probability of adversary's winning without sending a Hash query. For this, the adversary needs to forge $\operatorname{Send}\left(\operatorname{Dev}^{i}, \operatorname{Dev}^{j},<m_{1}>\right)$ and $\operatorname{Send}\left(\operatorname{Dev}^{j}, D e v^{i},<m_{2}>\right)$. Based on the difference lemma in consecutive games, the difference between $G_{3}$ and $G_{4}$ is insignificant. Thus,

$$
\left|\operatorname{Pr}\left[\operatorname{Succ}_{4}^{\mathcal{A}}(t)\right]-\operatorname{Pr}\left[\operatorname{Succ}_{3}^{\mathcal{A}}(t)\right]\right| \leq \frac{q_{s}}{2 \lambda}
$$

Game $\boldsymbol{G}_{\mathbf{5}}$. This game aims at breaking perfect forward secrecy using $\operatorname{Corrupt}(\operatorname{Dev}) \cdot \mathcal{A}$ tries to obtain $\operatorname{Dev}^{\prime} \mathrm{s}$ temporal secret parameters. The probability of adversary's winning the game is undistinguishable from the previous game as

$$
\left|\operatorname{Pr}\left[\operatorname{Succ}_{5}^{\mathcal{A}}(t)\right]-\operatorname{Pr}\left[\operatorname{Succ}_{4}^{\mathcal{A}}(t)\right]\right| \leq q_{h} A d v_{E C C D H P}^{\mathcal{A}}(t)+\frac{q_{h}^{2}}{2 \lambda}
$$

Game $\boldsymbol{G}_{\mathbf{6}}$. This game is over when the adversary obtains the original ID of oracle Dev by issuing a TestID(Dev) query. $\mathcal{A}$ needs to calculate $T I D_{i}=h\left(I D_{i} \| x\right), \alpha_{i}=x Q_{j}$, and $A I D_{i}=h\left(\alpha_{i}\right) \oplus T I D_{i}$. Assuming the nonce $x$, the difference between this game and the previous one is

$$
\left|\operatorname{Pr}\left[\operatorname{Succ}_{6}^{\mathcal{A}}(t)\right]-\operatorname{Pr}\left[\operatorname{Succ}_{5}^{\mathcal{A}}(t)\right]\right| \leq \frac{q_{h}^{2}}{2 \lambda}+\frac{q_{s}}{\lambda}
$$

Game $\boldsymbol{G}_{7}$. It simulates a situation in which the adversary issues a TestSK(Dev) query. This requires a successful implementation of SKReveal(Dev). The oracle flips the coin $c$. If $c=1$, it returns the session key. Using Hash 
query at most probability $\frac{q_{h}^{2}}{2 \lambda}$ and TestSK query at most probability $\frac{q_{s}}{\lambda}$, the difference between this game and the previous one is

$$
\left|\operatorname{Pr}\left[\operatorname{Succ}_{7}^{\mathcal{A}}(t)\right]-\operatorname{Pr}\left[\operatorname{Succ}_{6}^{\mathcal{A}}(t)\right]\right| \leq \frac{q_{h}^{2}}{2 \lambda}+\frac{q_{s}}{\lambda}
$$

The adversary's advantage to break security of the proposed protocol by guessing the coin inside TestSK query is $\operatorname{Adv}_{\mathcal{P}}^{\mathcal{A}}(t)=2 \operatorname{Pr}\left[c=c^{\prime}\right]-1$ where $c^{\prime}$ is the adversary's guess. Since the adversary is unable to distinguish real and random session keys, without issuing a Hash query with accurate entries we have $\operatorname{Pr}\left[\operatorname{Succ}_{7}^{\mathcal{A}}(t)\right]=$ $1 / 2$. Therefore, the theorem is proves based on formulas 3-9.

$$
\begin{gathered}
A d v_{\mathcal{P}}^{\mathcal{A}}(t) \leq 2 \times \sum_{G=1}^{6}\left|A d v_{G+1}^{\mathcal{A}}(t)-A d v_{G}^{\mathcal{A}}(t)\right| \leq \frac{q_{h}^{2}+\left(q_{S}+q_{e}\right)^{2}}{2 \lambda}+\frac{q_{s}}{2 \lambda}+q_{h}\left(A d v_{E C C D H P}^{\mathcal{A}}(t)\right)+\frac{q_{h}^{2}}{2 \lambda}+\frac{q_{h}^{2}}{2 \lambda}+\frac{q_{S}}{\lambda}+\frac{q_{h}^{2}}{2 \lambda}+ \\
\frac{q_{s}}{\lambda} \leq \frac{5 q_{S}+4 q_{h}^{2}+\left(q_{s}+q_{e}\right)^{2}}{2 \lambda}+q_{h}^{2} A d v_{E C C D H P}(t)
\end{gathered}
$$

Game $\boldsymbol{G}_{\mathbf{8}}$. The adversary implements Compromise (Dev) to obtain all static and dynamic secret parameters of $\mathrm{Dev}$. The aim is to perform a privileged-insider attack and compromise the whole network. In fact, by capturing one device, $\mathcal{A}$ seeks to capture the whole network. Since oracle instances are independent from each other, the probability of seizing one instance is independent from others. Thus, the adversary needs to compromise all oracle instances to capture the whole network. Therefore, his chance is trivial as follows:

$$
A d v_{N e t}^{\mathcal{A}}(t)=\prod_{i=1}^{\mathfrak{T}} A d v_{\mathcal{P}(i)}^{\mathcal{A}}(t)
$$

\subsection{Formal security analysis by BAN logic}

Table 3 shows signs and symbols of BAN logic [20], and the following rules are used in the BAN logic to prove mutual authentication between two IoT devices $D_{i}$ and $D_{j}$.

Table 3. Definition of BAN logic notations.

\begin{tabular}{|c|l|c|l|}
\hline Symbol & \multicolumn{1}{|c|}{ Description } & Symbol & \multicolumn{1}{|c|}{ Description } \\
\hline$P, Q$ & Entities in the authentication phase & $P \triangleleft X$ & $P$ has received $X$ \\
\hline$\#(X)$ & The formula $X$ is fresh & $P \mid \sim X$ & $P$ once sent $X$ \\
\hline$P \mid \equiv X$ & The entity $P$ believes $X$ & $<X>_{Y}$ & $X$ is XORed by $Y$ \\
\hline$P \Rightarrow X$ & $P$ has jurisdiction on $X$ & $(X)_{Y}$ & $X$ is hashed by $Y$ \\
\hline$P \stackrel{K}{\leftrightarrow} Q$ & Shared secret $K$ between $P$ and $Q$ & $\{X, Y\}_{K}$ & formulas $X$ and $Y$ encrypted by $K$ \\
\hline
\end{tabular}

- (R1) Message-Meaning: $\quad \frac{P \mid \equiv P \leftrightarrow Q, P \triangleleft\{X\}_{K}}{P \mid \equiv(Q \mid \sim X)}$

- (R3) Jurisdiction:

$\frac{P|\equiv(Q \Rightarrow X), P| \equiv(Q \mid \equiv X)}{P \mid \equiv X}$

- (R5) Belief:

$$
\frac{P|\equiv Q| \equiv(X, Y)}{P|\equiv Q| \equiv(X)}, \frac{P|\equiv(X), P| \equiv(Y)}{P \mid \equiv(X, Y)}
$$

- (R2) Nonce-Verification: $\frac{P|\equiv \#(X), P| \equiv(Q \mid \sim X)}{P \mid \equiv(Q \mid \equiv X)}$

- (R4) Freshness:

$\frac{P \mid \equiv \#(X)}{P \mid \equiv \#(X, Y)}$

The proposed protocol is based on following assumptions.

- $A 1: D_{i}\left|\equiv \#(x), D_{i}\right| \equiv \#\left(t_{i}\right), D_{i} \mid \equiv \#\left(t_{j}\right)$

- $A 2: D_{j}\left|\equiv \#(y), D_{j}\right| \equiv \#\left(t_{j}\right), D_{j} \mid \equiv \#\left(t_{i}\right)$

- $A 3: D_{i} \mid \equiv D_{i} \stackrel{M_{i}}{\leftrightarrow} T A$
- $A 5: D_{i} \mid \equiv D_{i} \stackrel{S K_{i j}}{\longleftrightarrow} D_{j}$

- $A 6: D_{j} \mid \equiv D_{i} \stackrel{S K_{j i}}{\longleftrightarrow} D_{j}$

- $A 7: D_{i}\left|\equiv D_{j} \Rightarrow D_{i} \stackrel{S K_{i j}}{\longleftrightarrow} D_{j}, D_{i}\right| \equiv D_{j} \Rightarrow M_{j}$ 
- $\quad A 4: D_{j} \mid \equiv D_{j} \stackrel{M_{j}}{\leftrightarrow} T A$

- $\quad A 8: D_{j}\left|\equiv D_{i} \Rightarrow D_{i} \stackrel{S K_{i j}}{\longleftrightarrow} D_{j}, D_{j}\right| \equiv D_{i} \Rightarrow M_{i}$

The process of mutual authentication aims to obtain the following goals

- $\quad G 1: D_{j}\left|\equiv D_{i}\right| \equiv U_{i} \stackrel{S K_{j i}}{\longleftrightarrow} D_{j}$

- $\quad G 2: D_{j} \mid \equiv D_{i} \stackrel{S K_{i j}}{\longleftrightarrow} D_{j}$
- $G 3: D_{i}\left|\equiv D_{j}\right| \equiv D_{i} \stackrel{S K_{i j}}{\longleftrightarrow} D_{j}$

- $G 4: D_{i} \mid \equiv D_{i} \stackrel{S K_{j i}}{\longleftrightarrow} D_{j}$

Messages are transmitted between $D_{i}$ and $D_{j}$ in a public channel. The idealized message has the following specifications

$$
M 1: D_{j} \triangleleft\left\{\left\langle T I D_{i}>_{\alpha_{i}}, x G,\left(T I D_{i}, \tau_{i}, t_{i}\right)_{M_{i} Q_{j}}, t_{i}\right\}, M 2: D_{i} \triangleleft\left\{<T I D_{j}>_{\beta_{j}}, y G,\left(T I D_{j},\left(T I D_{i}, T I D_{j}, t_{j}\right)_{K}\right)_{\left(M_{j} Q_{i}\right)}, t_{j}\right\}\right.
$$

Based on $A 4$, message $M 1$, applying the message-meaning rule $R 1$, and the fact that $D_{j}$ receives the first message, we have

$$
S 1: D_{j}\left|\equiv D_{i}\right| \sim\left(T I D_{i}, \alpha_{i}, \tau_{i}\left(T I D_{i}, \tau_{i}, t_{i}\right)_{M_{i} Q_{j}}, t_{i}\right)
$$

Using $A 2, S 1$, the freshness rule $R 4$, and the nonce verification rule $R 2$, we have

Based on $A 6, S 2$, and message $M 2$, we have

$$
S 2: D_{j}\left|\equiv D_{i}\right| \equiv\left(T I D_{i}, \alpha_{i}, \tau_{i}\left(T I D_{i}, \tau_{i}, t_{i}\right)_{M_{i} Q_{j}}, t_{i}\right)
$$

$$
S 3: D_{j}\left|\equiv D_{i}\right| \equiv\left(T I D_{i}, T I D_{j}, M_{i} Q_{j}, t_{i}\right)
$$

We achieve the first goal by applying the belief rule $R 5$ on $S 3$.

$$
G_{1}: D_{j}\left|\equiv D_{i}\right| \equiv\left(D_{i} \stackrel{S K_{j i}}{\longleftrightarrow} D_{j}\right)
$$

The second goal is achieved based on $A 8$, the jurisdiction rule $R 3$ and $S 3$.

$$
G_{2}: D_{j} \mid \equiv D_{i} \stackrel{S K_{i j}}{\longleftrightarrow} D_{j}
$$

Based on receiving the message $M 2$ at $D_{j}, A 3$ and $M 2$, we have

$$
S 4: D_{i}\left|\equiv D_{j}\right| \sim\left(T I D_{j}, \beta_{j}, \tau_{j},\left(D_{i} \stackrel{S K_{j i}}{\longleftrightarrow} D_{j}\right)_{\left(M_{j} Q_{i}\right)}, t_{j}\right)
$$

Using $A 1, S 4$, the message freshness, and the nonce verification, we achieve $S 5$.

According to $A 5$, ad $S 5$, we achieve the third goal.

$$
S 5: D_{i}\left|\equiv D_{j}\right| \equiv\left(T I D_{j}, \beta_{j}, \tau_{j},\left(D_{i} \stackrel{S K_{j i}}{\longleftrightarrow} D_{j}\right)_{\left(M_{j} Q_{i}\right)}, t_{j}\right)
$$

$$
G_{3}: D_{i}\left|\equiv D_{j}\right| \equiv D_{i} \stackrel{S K_{i j}}{\longleftrightarrow} D_{j}
$$

Eventually, based on $A 7, R 3$, and by assuming the correctness of the third goal, we achieve the fourth goal.

\subsection{Scyther tool}

$$
G_{4}: D_{i} \mid \equiv D_{i} \stackrel{S K_{j i}}{\longleftrightarrow} D_{j}
$$

Scyther tool is widely used for analyzing security protocols [21,22]. In this section, we show that the proposed protocol meets security claims like "Alive", "Nisynch", "Niagree", "weakagree", and "secret" for aliveness, non-injective synchronization, non-injective agreement, minimum agreement, and confidentiality, respectively. Moreover, confidentiality of session key is preserved and IoT device IDs remain secret. Scyther code and evaluation results are given below in Fig 9 and Fig 10. 


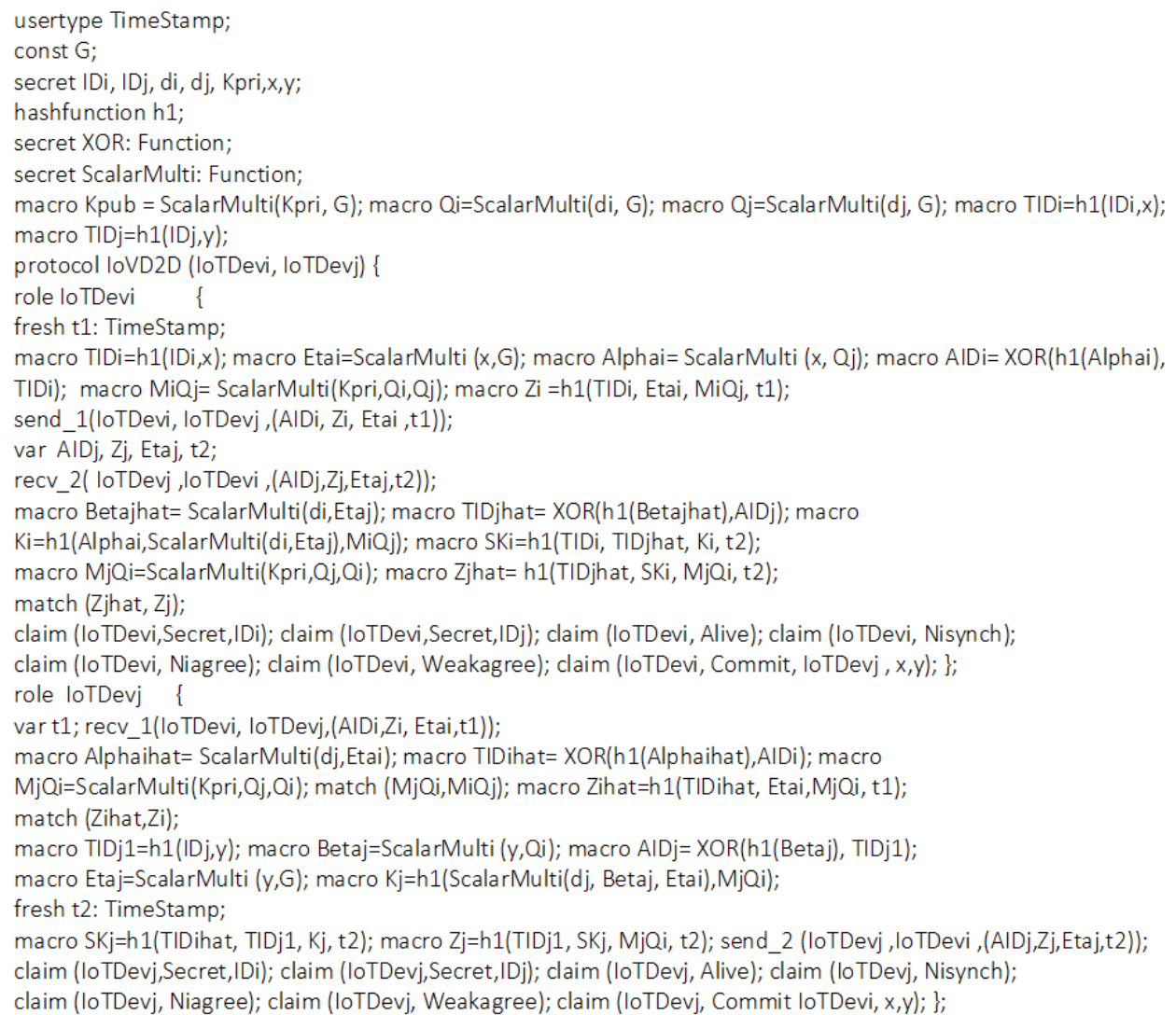

\section{Fig .9. Scyther code of the proposed protocol}




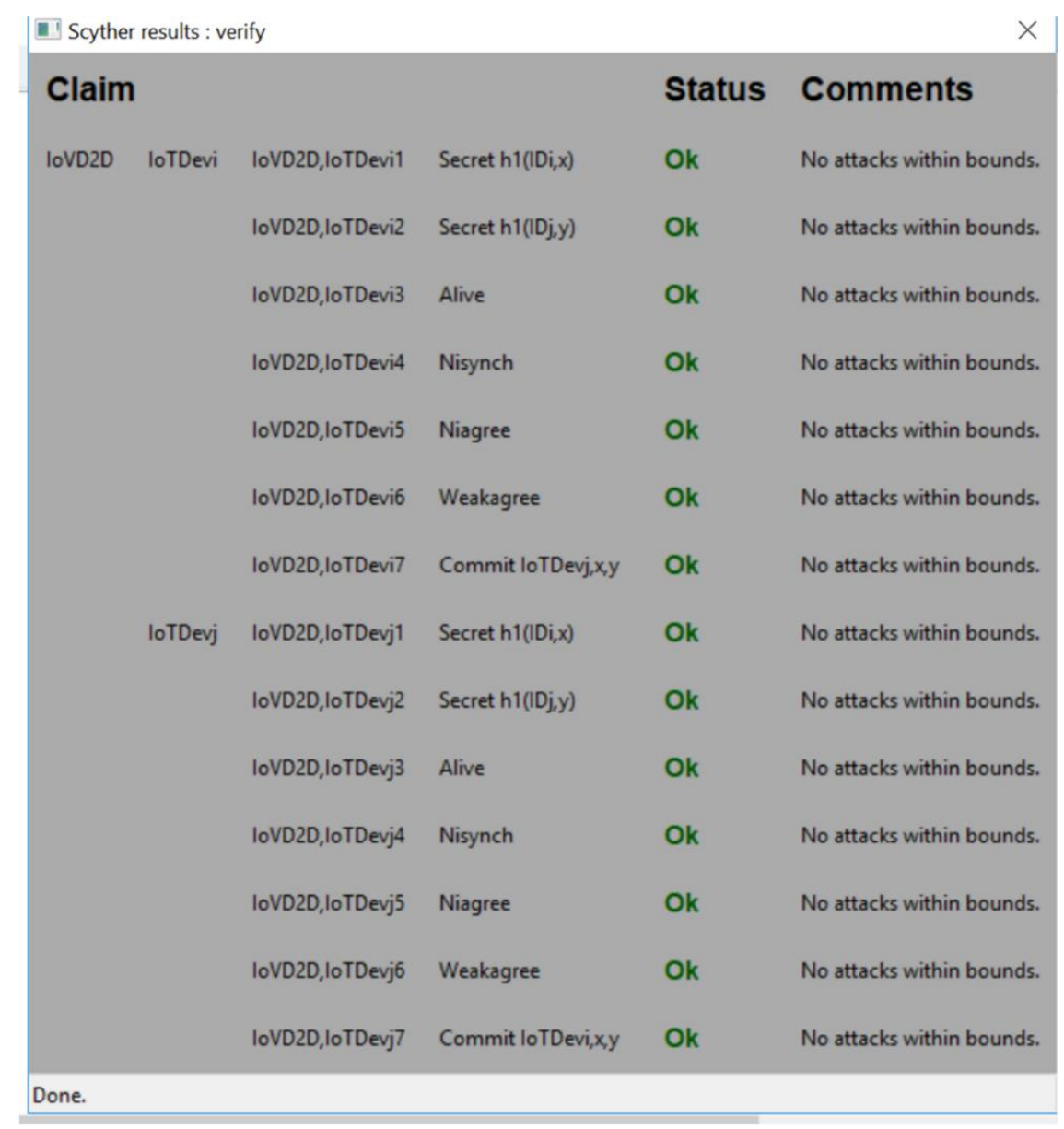

Fig .10. Security analysis of the proposed protocol using Scyther tool

As seen in Fig $10, I D_{i}, I D_{j}, T I D_{i}=h\left(I D_{i} \| x\right)$, and $T I D_{i}=h\left(I D_{i} \| y\right)$ are secure against intruder attacks and their secrecy is preserved. Thus, the proposed protocol is highly anonymous and untraceable.

\subsection{Informal security analysis}

It is proved in this section that the proposed protocol provides security measures for D2D key agreement protocol. Table 4 compares the proposed protocol with other existing protocols in features and resisting known attacks.

5.3.1. Replay Attack. The protocol uses timestamps $t_{1}$ and $t_{2}$, random-temporary nonces $x$ and $y$, that are used as hashed values $Z_{i}=h\left(\operatorname{TID}_{i}\left\|\tau_{i}\right\| M_{i} Q_{j} \| t_{1}\right)$. Any changes in them violates message integrity and the condition $Z_{i}=$ ? $Z_{i}^{\prime}=h\left(T I D_{i}^{\prime}\left\|\tau_{i}\right\| M_{j} Q_{i} \| t_{1}\right)$ will no longer hold. Hence it is secure against replay attack because all the messages are sent in a valid $\Delta T$ time period.

5.3.2. Man-In-The-Middle (MITM) Attack. It is assumed that adversary $\mathcal{A}$ has access to $I D_{i}$ and $I D_{j}$ of IoT devices. He seeks to seize and manipulate messages $<A I D_{i}, Z_{i}, \tau_{i}, t_{1}>$ and $<A I D_{j}, Z_{j}, \tau_{j}, t_{2}>$ to IoT devices and forge an authorized device. Since the adversary does not have long secret key $d_{i}$ and $d_{j}$, he cannot retrieve $\alpha_{i}=d_{j} \tau_{i}$ and $\beta_{j}=d_{i} \tau_{j}$, and is unable to perform the man-in-the-middle attack.

5.3.3. Privilege-Insider Attack. An unauthorized insider IoT device cannot seize device IDs or session keys in the network pseudo-identity $A I D_{i}=h\left(\alpha_{i}\right) \oplus T I D_{i}$ are sent to the channel where $\alpha_{i}=x Q_{j}$ and only $D_{j}$ has access 
to $\operatorname{TI}_{i}$ because $\alpha_{i}$ is retrieved as $\alpha_{i}=d_{j} \tau_{i}$. This prevents all unauthorized insider access to other session keys and device IDs. While Alzahrani et al.'s [6] protocol cannot withstand the privileged insider attack.

5.3.4. Device capture attack. If an adversary $\mathcal{A}$ captures an IoT device and extract its sensitive information by power analysis techniques to achieve $M_{i}$ and $I D_{i}$, he cannot get other devices' information such as secret stored value $M_{j}$, private key $d_{j}$, and original identity $I D_{j}$. In addition, if private key $d_{i}$ is not stored in $D_{i}$, the adversary cannot compromise that node. Thus, the proposed protocol is secure against device compromise attack, while $[6,7,8,9]$ are insecure against that.

5.3.5. Known Specific Temporary Information Attack. If an adversary $\mathcal{A}$ obtains secret parameters $x$ and $y$, he cannot get session key $S K_{i j}=h\left(T I D_{i}\left\|T I D_{j}\right\| K \| t_{2}\right)$ because hidden value $K$ is $K=h\left(\alpha_{i}\left\|d_{i} \tau_{j}\right\| M_{i} Q_{j}\right)=$ $h\left(d_{j} \tau_{i}\left\|\beta_{j}\right\| M_{j} Q_{i}\right)$ wherein $M_{i}$ and $M_{j}$ are stored in each device and is inaccessible. Therefore, our protocol is secure against temporary secret leakage attack.

5.3.6. Key Compromise Impersonation (KCI) Attack. Once an adversary $\mathcal{A}$ gains access to private keys $d_{i}$ or $d_{j}$, he is unable to get session key $S K_{i}=S K_{j}$ because hidden parameters $M_{i} / M_{j}$ are stores in devices as $M_{i}=K_{p r i} Q_{i}$ which is extracted only when the Elliptic Curve Diffie-Hellman (ECDHP) Problem is solved. Thus, the proposed protocol is secure against KCI attack.

5.3.7. Anonymity and Untraceability. Messages are transmitted between two devices with pseudo-temporary identity $A I D_{i}=h\left(\alpha_{i}\right) \oplus T I D_{i}$ and $A I D_{j}=h\left(\beta_{j}\right) \oplus T I D_{j}$ where $T I D_{i}$ and $T I D_{j}$ are temporary IDs. If an adversary $\mathcal{A}$ obtains private keys $d_{i}$ and $d_{j}$, he can only gain access to the temporary, not permanent, IDs. Moreover, earlier messages cannot be retrieved because temporary IDs change in each authentication session. Thus, the proposed protocol is anonymous and untraceable. In other words, it provides dual anonymous communication.

5.3.8. Perfect Forward/Backward Secrecy. If an adversary $\mathcal{A}$ obtains the session key in a session, he will not have access to other session keys in other session because session keys are computed independently, including random nonces ( $x$ and $y$ ) and temporary $T I D_{i}$ and $T I D_{j}$ that change in each authentication session. Therefore, the proposed protocol preserves perfect forward backward secrecy.

5.3.9. Impersonation Attack. To successfully perform this attack and forge IoT devices, the adversary needs to duplicate messages $Z_{i}=h\left(T I D_{i}\left\|\tau_{i}\right\| M_{i} Q_{j} \| t_{1}\right)$ and $Z_{j}=h\left(T I D_{j}\left\|S K_{j}\right\| t_{2}\right)$ to be certified by the shared protocol. An adversary can never generate a valid message to forge an authorized device in the network because it does not have access to private key $d_{i}$ and original $I D_{i}$. Our proposed protocol is able to withstand impersonation attack.

5.3.10. Known-Key Attack. When an IoT device shares a secure session key with another device in the network, an adversary $\mathcal{A}$ who seizes the key cannot obtain the keys of other devices because each key has its own specific parameters $x, y, K_{i}, K_{j}$, and temporary TID. To overcome this challenge, the adversary must solve Elliptic Curve Discrete Logarithm (ECDL) problem, ECDHP problem, and one-way hash function, which is practically infeasible.

5.3.11. Key Escrow Problem. If private key of TA is disclosed, other keys remain secret because only TA is involved in key generation process and the private key $d_{i}=f_{i}+r_{i}=\left(e_{i}+P_{i}\right) K_{p r i}+r_{i}$ consists of random nonce $r_{i} \in Z_{p}^{*}$ that is unknown to TA. Therefore, the proposed protocol solves key escrow problem and single point of failure.

5.3.12. Self-Certification Mechanism. Each IoT device $D_{i}$ stores $M_{i}=K_{p r i} Q_{i}$ in its memory in registration phase, and does not need an online trusted third-party TA in authentication and key agreement phase. Therefore, our protocol solves key escrow problem, single-point of failure, and has a great performance in certificate generation and verification process. 
5.3.13. Clogging Attack. The adversary cannot perform clogging attack and waste IoT resources because both agents mutually authenticate each other before sharing a session key. however, Li et al.'s and Chaudhry et al.'s protocols are insecure against this attack.

5.3.14. Other features. Many protocols neglect updating long secret credentials. Nevertheless, our protocol offers a secure authentication and key agreement session between IoT devices while updating paired private and public keys of each device in the presence of a TA in a pubic channel. This increases its applicability. Moreover, registration phase of this protocol is done in an insecure channel that increases its scalability.

Table 4. Security Comparisons

\begin{tabular}{|c|c|c|c|c|c|c|}
\hline \multirow{2}{*}{\multicolumn{2}{|c|}{ Comparative point }} & \multicolumn{5}{|c|}{ protocols } \\
\hline & & $\begin{array}{l}\text { Chaudhry } \\
\text { et al. [10] }\end{array}$ & $\begin{array}{l}\text { Das et al. } \\
{[18]}\end{array}$ & $\begin{array}{l}\text { Alzahrani } \\
\text { et al. [6] }\end{array}$ & $\begin{array}{l}\text { Li et al. } \\
\text { [9] }\end{array}$ & $\begin{array}{c}\text { Our } \\
\text { protocol }\end{array}$ \\
\hline \multirow{7}{*}{ Features } & Mutual authentication & $\checkmark$ & $\checkmark$ & $\checkmark$ & $x$ & $\checkmark$ \\
\hline & Perfect forward secrecy & $x$ & $\checkmark$ & $\checkmark$ & $\checkmark$ & $\checkmark$ \\
\hline & $\begin{array}{l}\text { IoT device anonymity and } \\
\text { untraceability }\end{array}$ & $x$ & $x$ & $x$ & $x$ & $\checkmark$ \\
\hline & Long secret keys updating & $x$ & $x$ & $x$ & $x$ & $\checkmark$ \\
\hline & $\begin{array}{l}\text { Using public channel in registration } \\
\text { and updating keys phase }\end{array}$ & $x$ & $x$ & $x$ & $x$ & $\checkmark$ \\
\hline & Security proof & $\begin{array}{l}\text { ROM } \\
\text { model }\end{array}$ & $\begin{array}{c}\text { ROR } \\
\text { model }\end{array}$ & $\begin{array}{c}\text { ROM } \\
\text { model, } \\
\text { BAN logic }\end{array}$ & $\begin{array}{l}\text { ROM } \\
\text { model }\end{array}$ & $\begin{array}{c}\text { ROR } \\
\text { model, } \\
\text { BAN logic }\end{array}$ \\
\hline & Security analysis & - & $\begin{array}{l}\text { AVISPA } \\
\text { tool }\end{array}$ & - & - & $\begin{array}{l}\text { Scyther } \\
\text { tool }\end{array}$ \\
\hline \multirow{11}{*}{$\begin{array}{c}\text { Resistance } \\
\text { to }\end{array}$} & Strong replay attack & $\checkmark$ & $\checkmark$ & $\checkmark$ & $x$ & $\checkmark$ \\
\hline & Clogging attack & $x$ & $\checkmark$ & $\checkmark$ & $x$ & $\checkmark$ \\
\hline & Desynchronization attack & $x$ & $\checkmark$ & $\checkmark$ & $x$ & $\checkmark$ \\
\hline & Man-in-the-Middle attack & $x$ & $x$ & $\checkmark$ & $\checkmark$ & $\checkmark$ \\
\hline & IoT device impersonation attack & $\checkmark$ & $x$ & $\checkmark$ & $\checkmark$ & $\checkmark$ \\
\hline & $\begin{array}{lcr}\text { IoT deice Key } & \text { compromise } \\
\text { Impersonation attack } & \\
\end{array}$ & $\checkmark$ & $\checkmark$ & $x$ & $x$ & $\checkmark$ \\
\hline & IoT device capture attack & $\checkmark$ & $\checkmark$ & $\checkmark$ & $\checkmark$ & $\checkmark$ \\
\hline & Privilege-insider attack & $\checkmark$ & $\checkmark$ & $x$ & $\checkmark$ & $\checkmark$ \\
\hline & $\begin{array}{l}\text { Known specific temporary information } \\
\text { attack }\end{array}$ & $\checkmark$ & $\checkmark$ & $\checkmark$ & $\checkmark$ & $\checkmark$ \\
\hline & Key escrow problem & $\checkmark$ & $\checkmark$ & $\checkmark$ & $\checkmark$ & $\checkmark$ \\
\hline & Known key attack & $\checkmark$ & $\checkmark$ & $\checkmark$ & $\checkmark$ & $\checkmark$ \\
\hline
\end{tabular}

$\checkmark:$ Yes, $\mathbf{x}:$ No

Based on Table 4 and other analyses, our protocol has a perfect security profile while other protocols [6, 9, 10, 18] suffer from flaws. Thus, our proposed protocol is better than others for authentication of D2D communications.

\subsection{Analysis of efficiency}

Our protocol is compared with other existing protocols $[6,9,10,18]$ in terms of communication and computational overhead. We assume that timestamp, ID, nonce, hash value and elliptical curve point are 32, $128,128,160$, and 320 bits, respectively. The time for hash operation $\left(T_{h}\right)$, symmetric encryption/decryption 
$\left(T_{e n c / d e c}^{s}\right)$, point addition $\left(T_{p a}\right)$, and point scalar multiplication on elliptic curve $\left(T_{p m}\right)$ is $0.00032,0.0056$, 0.0044 , and $0.0171 \mathrm{sec}[23,18]$. Computation and communication costs of our protocol are $0.14128 \mathrm{sec}$ and 1344 bits, respectively. Table 5 compares our protocol with existing ones in terms of communication and computation overheads. It shows that the proposed protocol has lower computational overhead than other related protocols. However, it resists KCI attack, replay attack, insider attack, clogging attack, and provides anonymity (see Table 4). In [9], only one session key is shared and no authentication mechanism is applied. Security is an essential factor in IoT networks and overhead pays its due for that. Our protocol is compared with others in terms of computational overhead and communication overhead in Fig 11.

Table 5. Comparison of Communication and Computation Overheads

\begin{tabular}{|l|l|c|}
\hline Protocol & Communication Cost & Computation Cost \\
\hline Alzahrani et al. [6] & $4 \mid$ ECC $|+3|$ Hash $|+2|$ Timestamp $\mid$ & $8 T_{p m}+2 T_{p a}+8 T_{h}$ \\
\hline Li et al. [9] & $2|I D|+4 \mid$ ECC $\mid$ & $12 T_{p m}+2 T_{p a}+6 T_{h}$ \\
\hline Chaudhry et al. [10] & $2|I D|+6 \mid$ ECC $|+2|$ Hash $|+3|$ Timestamp $\mid$ & $10 T_{p m}+6 T_{p a}+8 T_{h}$ \\
\hline Das et al. [18] & $2 \mid$ ID $|+8|$ ECC $|+2|$ Hash $|+3|$ Timestamp $\mid$ & $18 T_{p m}+6 T_{p a}+12 T_{h}$ \\
\hline Our protocol & $2 \mid$ ECC $|+4|$ Hash $|+2|$ Timestamp $\mid$ & $8 T_{p m}+14 T_{h}$ \\
\hline
\end{tabular}

$|x|$ : Bit length of variable $x, T_{x}$ : execution time for the operation $x$.
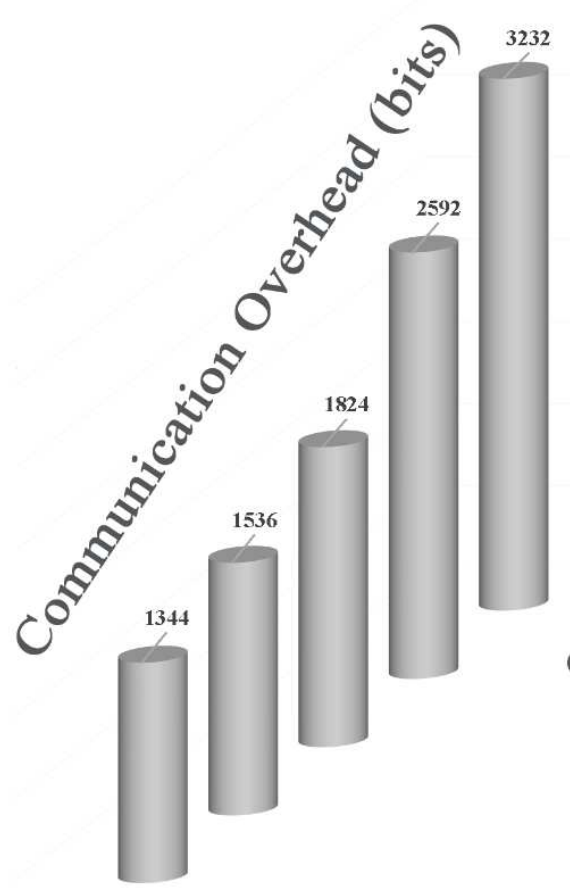

Das et al.[18]

Chaudhry et al. [10]

Alzahrani et al. [6]

Li et al. [9]

Our Protocol

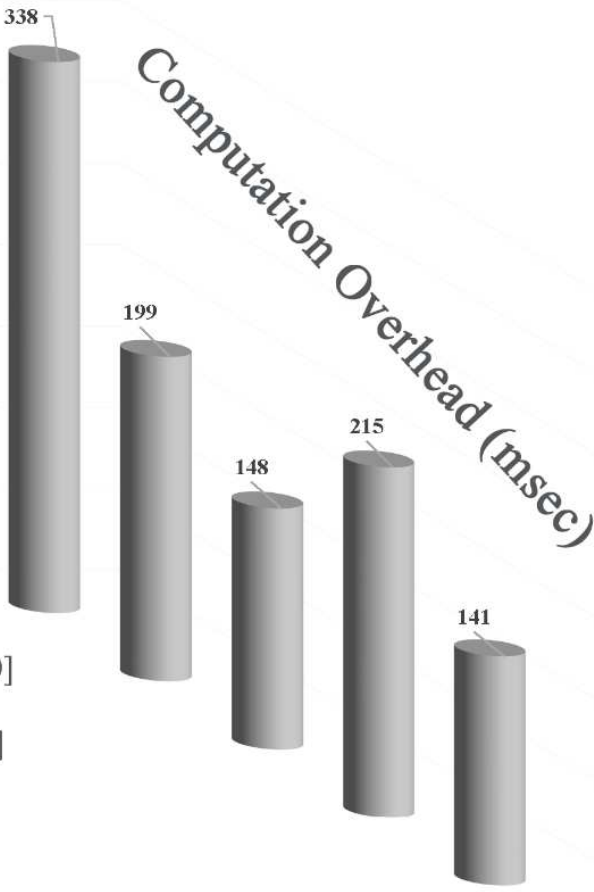

Fig .11. Communication and communication overhead comparison.

The performance of the proposed protocol, compared to existing protocols, in terms of energy consumption for 500 authentication and key agreement sessions for IoT devices in a $100 \times 100 \mathrm{~m}^{2}$ environment is simulated in Matlab R2017a. Results are shown in Fig. 12. Increased number of sessions leads to increase energy 
consumption in [10] and [18]. But the proposed protocol offers the most optimal performance because energy management in ubiquity 6G networks is important along with security issues. Our protocol improves performance to $20-65 \%$.

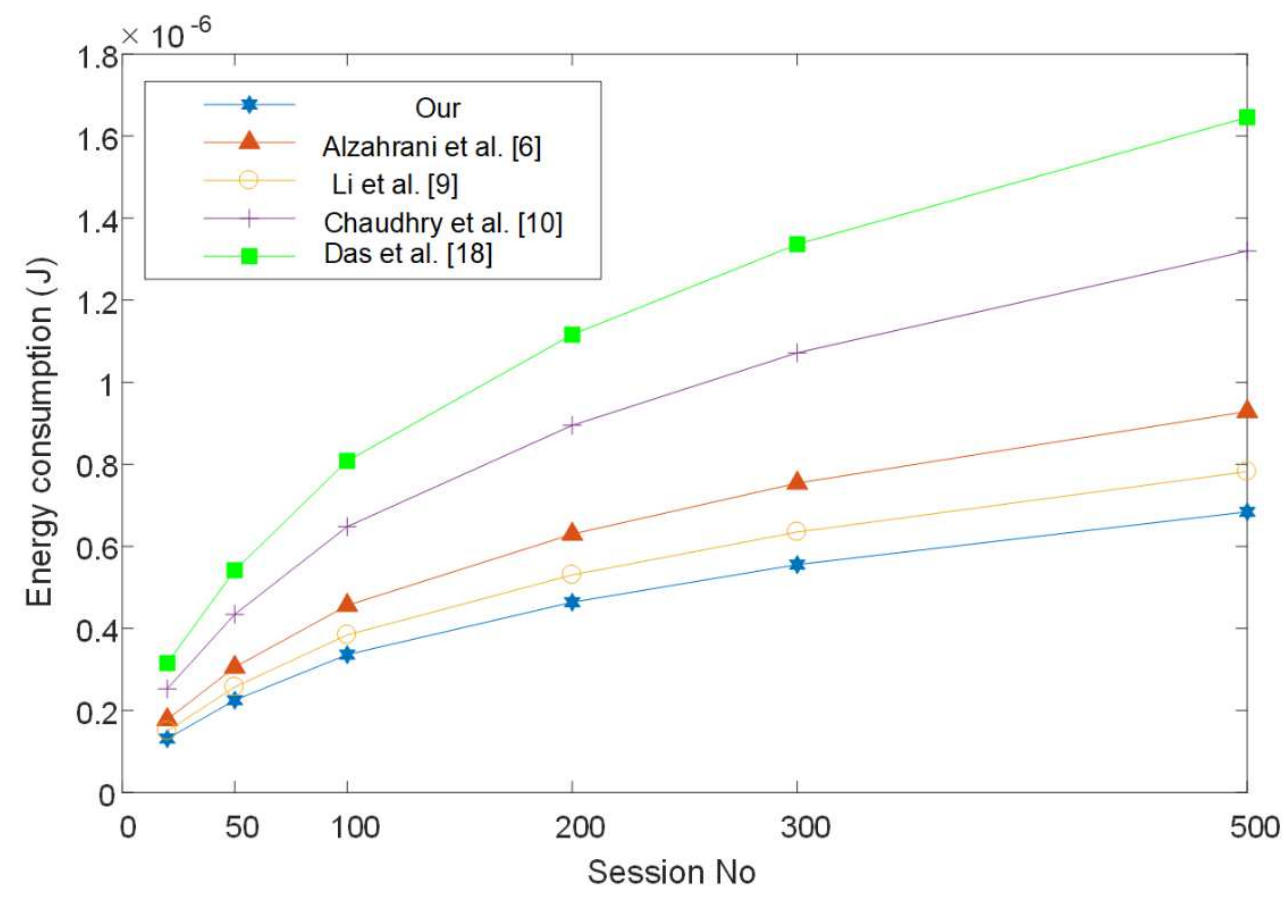

Fig. 12. Energy consumption comparison.

\section{Conclusion}

Widespread use of IoT technologies and lack of comprehensive standard protocols for IoT environments call for new authentication and key agreement protocols. The present study examined the flaws of mutual authentication and key agreement protocols for D2D communications in IoT networks. It also proposed a new protocol include four phases: system initialization, IoT device registration, authentication and key agreement, key pair updating. Our protocol could overcome security issues and resist well-known attacks such as KCI attack, replay attack, insider attack, and provide strong anonymity. Also, it used public channels in IoT device registration and public/private key updating phases. BAN logic, ROR model, and Scyther tool were used for security analysis. The protocol performance was also compared with other protocols in terms of computational, communication overhead, and energy consumption and showed to have a better performance than other compared protocols. Finally, a blockchain-based group authentication protocol in D2D communications while provides anonymity is proposed for future work.

\section{Compliance with ethical standards}

Conflict of interest. The authors declare that they have no conflict of interest.

Ethical approval. This article does not contain any studies with human participants or animals performed by any of the authors.

Informed consent. Informed consent was obtained from all individual participants included in the study. 


\section{Author contributions}

All authors contributed to the study conception and design. Material preparation, data collection and analysis were performed by Rahman Hajian, Abbas Haghighat, and Seyed Hossein Erfani. All authors read and approved the final manuscript.

\section{References}

[1] Saad, W., Bennis, M., \& Chen, M. (2019), A vision of 6G wireless systems: Applications, trends, technologies, and open research problems, IEEE network, 1-9, https://doi.org/.10.1109/MNET.001.1900287.

[2] Wang, M., Yan, Z., \& Niemi, V. (2017), UAKA-D2D: Universal authentication and key agreement protocol in D2D communications. Mobile Networks and Applications, 22(3), 510-525, https://doi.org/10.1007/s11036017-0870-5.

[3] Seok, B., Sicato, J. C. S., Erzhena, T., Xuan, C., Pan, Y., \& Park, J. H. (2020), Secure D2D Communication for 5G IoT Network Based on Lightweight Cryptography, Applied Sciences, 10(1), 217, https://doi.org/10.3390/app10010217.

[4] Hajian, R., \& Erfani, S. H. (2020). CHESDA: continuous hybrid and energy-efficient secure data aggregation for WSN. The Journal of Supercomputing, 1-31, https://doi.org/10.1007/s11227-020-03455-z.

[5] Banerjee, S., Odelu, V., Das, A. K., Srinivas, J., Kumar, N., Chattopadhyay, S., \& Choo, K. K. R. (2019), A Provably Secure and Lightweight Anonymous User Authenticated Session Key Exchange Scheme for Internet of Things Deployment, IEEE Internet of Things Journal, 6(5), 8739-8752, https://doi.org/10.1109/JIOT.2019.2923373.

[6] Alzahrani, B. A., Chaudhry, S. A., Barnawi, A., Al-Barakati, A., \& Shon, T. (2020), An Anonymous Device to Device Authentication Protocol Using ECC and Self Certified Public Keys Usable in Internet of Things Based Autonomous Devices, Electronics, 9(3), 520, https://doi.org/10.3390/electronics9030520.

[7] Islam, S. H., \& Biswas, G. P. (2015), Design of two-party authenticated key agreement protocol based on ECC and self-certified public keys, Wireless Personal Communications, 82(4), 2727-2750, https://doi.org/10.1007/s11277-015-2375-5.

[8] Mandal, S., Mohanty, S., \& Majhi, B., (2018), Cryptanalysis and enhancement of an anonymous self-certified key exchange protocol, Wireless Personal Communications, 99(2), 863-891, https://doi.org/10.1007/s11277017-5156-5.

[9] Li, Q., Hsu, C. F., Raymond Choo, K. K., \& He, D. (2019), A Provably Secure and Lightweight Identity-Based Two-Party Authenticated Key Agreement Protocol for Vehicular Ad Hoc Networks, Security and Communication Networks, 7871067, https://doi.org/10.1155/2019/7871067.

[10] Chaudhry, S. A., Yahya, K., Al-Turjman, F., \& Yang, M. H. (2020). A secure and reliable device access control scheme for IoT based sensor cloud systems. IEEE Access, 8, 139244-139254, https://doi.org/10.1109/ACCESS.2020.3012121.

[11] Amin, R., Islam, S. H., Biswas, G. P., Giri, D., Khan, M. K., \& Kumar, N., (2016), A more secure and privacyaware anonymous user authentication scheme for distributed mobile cloud computing environments, Security and Communication Networks, 9(17), 4650-4666,https://doi.org/10.1002/sec.1655.

[12] Das, A. K., Wazid, M., Kumar, N., Khan, M. K., Choo, K. K. R., \& Park, Y, (2017), Design of secure and lightweight authentication protocol for wearable devices environment, IEEE journal of biomedical and health informatics, 22(4), 1310-1322, https://doi.org/10.1109/】BHI.2017.2753464. 
[13] Simplicio Jr, M. A., Silva, M. V., Alves, R. C., \& Shibata, T. K, (2017), Lightweight and escrow-less authenticated key agreement for the internet of things. Computer Communications, 98, 43-51, https://doi.org/10.1016/i.comcom.2016.05.002.

[14] Wu, F., Xu, L., Li, X., Kumari, S., Karuppiah, M., \& Obaidat, M. S., (2018), A lightweight and provably secure key agreement system for a smart grid with elliptic curve cryptography, IEEE Systems Journal, 13(3), 28302838, https://doi.org/10.1109/ISYST.2018.2876226.

[15] Shuai, M., Liu, B., Yu, N., Xiong, L., \& Wang, C. (2020), Efficient and privacy-preserving authentication scheme for wireless body area networks, Journal of Information Security and Applications, 52, 102499, https://doi.org/10.1016/j.jisa.2020.102499.

[16] Lara, E., Aguilar, L., Sanchez, M. A., \& García, J. A. (2020). Lightweight Authentication Protocol for M2M Communications of Resource-Constrained Devices in Industrial Internet of Things, Sensors, 20(2), 501, https://doi.org/10.3390/s20020501.

[17] Islam, S. H. (2020), Provably secure two-party authenticated key agreement protocol for post-quantum environments. Journal of Information Security and Applications, 52, 102468, https://doi.org/10.1016/i.jisa.2020.102468.

[18] Das, A. K., Wazid, M., Yannam, A. R., Rodrigues, J. J., \& Park, Y. (2019). Provably secure ECC-based device access control and key agreement protocol for IoT environment. IEEE Access, 7, 55382-55397, https://doi.org/10.1109/ACCESS.2019.2912998.

[19] R. Vinoth, L. J. Deborah, P. Vijayakumar and N. Kumar, (2020), "Secure Multi-factor Authenticated Key Agreement Scheme for Industrial IoT," in IEEE Internet of Things Journal, https://doi: 10.1109/IIOT.2020.3024703.

[20] Burrows, Michael, Martin Abadi, and Roger Michael Needham. "A logic of authentication." Proceedings of the Royal Society of London. A. Mathematical and Physical Sciences, 426(1871), 233-271 (1989), https://doi.org/10.1098/rspa.1989.0125.

[21] C. Cremers, Scyther tool, (n.d.). http://www.cs.ox.ac.uk/people/cas.cremers/scyther/ (accessed June 13, 2018).

[22] Nikooghadam, M., \& Amintoosi, H. (2020). A secure and robust elliptic curve cryptography-based mutual authentication scheme for session initiation protocol. Security and Privacy, 3(1), e92, https://doi.org/10.1002/spy2.92.

[23] Srinivas, J., Das, A. K., Kumar, N., \& Rodrigues, J. (2018). Cloud centric authentication for wearable healthcare monitoring system. IEEE Transactions on Dependable and Secure Computing, https://doi.org/10.1109/TDSC.2018.2828306. 